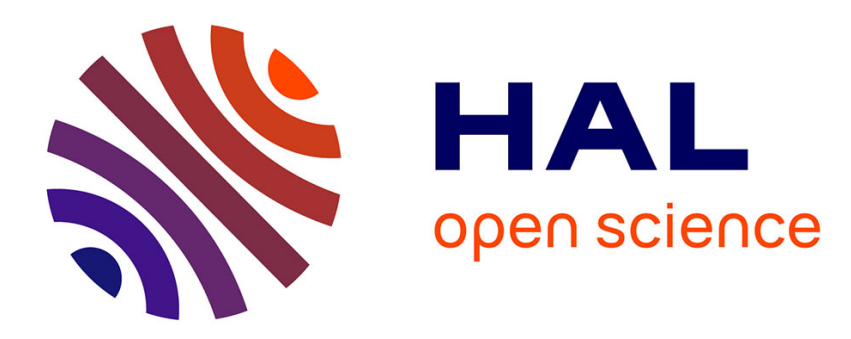

\title{
Numerical simulations for the dynamics of flexural shells
} Xiaoqin Shen, Luisa Piersanti, Paolo Piersanti

\section{To cite this version:}

Xiaoqin Shen, Luisa Piersanti, Paolo Piersanti. Numerical simulations for the dynamics of flexural shells. 2019. hal-02275020v2

\section{HAL Id: hal-02275020 \\ https://hal.science/hal-02275020v2}

Preprint submitted on 1 Sep 2019

HAL is a multi-disciplinary open access archive for the deposit and dissemination of scientific research documents, whether they are published or not. The documents may come from teaching and research institutions in France or abroad, or from public or private research centers.
L'archive ouverte pluridisciplinaire HAL, est destinée au dépôt et à la diffusion de documents scientifiques de niveau recherche, publiés ou non, émanant des établissements d'enseignement et de recherche français ou étrangers, des laboratoires publics ou privés. 


\section{Numerical simulations for the dynamics of flexural shells}

\author{
Xiaoqin Shen ${ }^{1}$, Luisa Piersanti ${ }^{2}$ and Paolo Piersanti ${ }^{3}$
}

\author{
Journal Title \\ $\mathrm{XX}(\mathrm{X}) 1+30$ \\ (C) The Author(s) 0000 \\ Reprints and permission: \\ sagepub.co.uk/journalsPermissions.nav \\ DOI: $10.1177 /$ ToBeAssigned \\ www.sagepub.com/
}

@SAGE

\author{
Xiaoqin Shen', Luisa Piersanti and Paolo Piersanti
}

\begin{abstract}
In this paper we study a model describing the displacement of a linearly elastic flexural shell subjected to given dynamic loads from the computational point of view. The model under consideration takes the form of a set of hyperbolic variational equations posed over the space of admissible linearized inextensional displacements, and a set of initial conditions. Since the original model is not suitable for the implementation of a finite element method, we conduct the experiments on the corresponding penalised model. It was recently shown that the solution to such a penalised model is a good approximation of the solution to the original model. Numerical tests are therefore conducted on the the penalised model; the approximation of the solution to the penalised model is obtained via Newmark's scheme, which is then implemented and tested for shells having the following middle surfaces: a portion of a cylinder, and a portion of a cone. For sake of completeness, we also present the results of the numerical tests related to a model describing the displacement of a linearly elastic elliptic membrane shell under the action of given dynamic loads.
\end{abstract}

\title{
Keywords
}

Flexural shells, Elliptic membrane shells, finite element method, Newmark's scheme, cylindrical shell, conical shell, spherical shell

\section{Introduction}

Flexural shells are widely used in many applicative fields such as physics, engineering and material science. Some remarkable applications involving the usage of such shells are: reinforced oil palm shell and palm oil clinker concrete (PSCC) beam [1], smart composite shell panels [2], functionally graded

\footnotetext{
${ }^{1}$ School of Sciences, Xi'an University of Technology, Xi'an, P.R. China

2 Department of Mathematics and Computer Science, University of Perugia, Perugia, Italy

3 Institute of Mathematics and Scientific Computing, Karl-Franzens-Universitat, Graz, Austria

Corresponding author:

Paolo Piersanti, Institute of Mathematics and Scientific Computing, Karl-Franzens-Universitat Graz, 36 Heinrichstrasse, Graz, Austria

Email: paolo.piersanti@uni-graz.at 
spherical shell panel [3], anisogrid lattice conical shells [4, and reinforced Eco-friendly coconut shell concrete [5]. Because of its wide range of applications, the theory of flexural shells is one of the most important branches in Mathematical Elasticity.

Unlike the static case, which was addressed by Ciarlet and his associates in the references [6], [7, 8], 9], 10], 11, [12, [13, [14, [15, [16, [17, 18, there are very few reference about the time-dependent case. In this direction we cite, for instance, the papers [19] and 20].

To our best knowledge, there are no references that treat the numerical simulation for wellestablished models describing the dynamics of flexural shells.

In Section 2 we present some geometrical and analytical background; in Section 3 we formulate the problem describing the displacement of a flexural shell when it is subjected to given dynamic loads; in Section 4 we formulate the corresponding penalised problem, which is easier to treat in a context of numerical simulations, we recall the result establishing the existence and uniqueness of the solution of the model under consideration, and we analyse the convergence of the solution of the penalised model to the solution of the original model; in Sections 5 and 6 , we rigorously state the algorithm that implements Newmark's scheme for the penalised problem and we discuss the convergence of the approximate solution it outputs to the solution of the original model; finally, in Sections 7, 8 and 9 we perform numerical experiments in the case where the middle surface of the linearly elastic shell under consideration is a portion of a cylinder, a portion of a cone, and a spherical cap, respectively.

\section{Geometrical preliminaries}

For details about the classical notions of differential geometry recalled in this section see, e.g., 21] or [22].

Greek indices, except $\varepsilon$ and $\nu$, take their values in the set $\{1,2\}$, while Latin indices, except when they are used for indexing sequences, take their values in the set $\{1,2,3\}$, and the summation convention with respect to repeated indices is systematically used in conjunction with these two rules. The notation $\mathbb{E}^{3}$ designates the three-dimensional Euclidean space; the Euclidean inner product and the vector product of $\boldsymbol{u}, \boldsymbol{v} \in \mathbb{E}^{3}$ are denoted $\boldsymbol{u} \cdot \boldsymbol{v}$ and $\boldsymbol{u} \wedge \boldsymbol{v}$; the Euclidean norm of $\boldsymbol{u} \in \mathbb{E}^{3}$ is denoted $|\boldsymbol{u}|$. The notation $\delta_{i}^{j}$ designates the Kronecker symbol.

Given an open subset $\Omega$ of $\mathbb{R}^{n}$, notations such as $L^{2}(\Omega), H^{m}(\Omega)$, or $H_{0}^{m}(\Omega), m \geq 1$, designate the usual Lebesgue and Sobolev spaces, and the notation $\mathcal{D}(\Omega)$ designates the space of all functions that are infinitely differentiable over $\Omega$ and have compact support in $\Omega$. The notation $\|\cdot\|_{X}$ designates the norm in a normed vector space $X$. The dual space of a vector space $X$ is denoted by $X^{*}$. Spaces of vector-valued functions are denoted with boldface letters. Lebesgue-Bochner spaces defined over a bounded open interval $I$ (cf. [23), are denoted $L^{p}(I ; H)$, where $H$ is a Banach space and $1 \leq p \leq \infty$. The notation $\|\cdot\|_{0, \Omega}$ designates the norm of the Lebesgue space $L^{2}(\Omega)$, and the notation $\|\cdot\|_{m, \Omega}$, designates the norm of the Sobolev space $H^{m}(\Omega), m \geq 1$. The notation $\|\cdot\|_{L^{p}(I ; H)}$ designates the norm of the Lebesgue-Bochner space $L^{p}(I ; H)$. The notations $\dot{\eta}$ and $\ddot{\eta}$ denote the first weak derivative with respect to $t \in I$ and second weak derivative with respect to $t \in I$ of a scalar function $\eta$ defined over the interval $I$. The notations $\dot{\boldsymbol{\eta}}$ and $\ddot{\boldsymbol{\eta}}$ denote the first weak derivative with respect to $t \in I$ and second weak derivative with respect to $t \in I$ of a vector-valued function $\boldsymbol{\eta}$ defined over the interval $I$.

A domain in $\mathbb{R}^{n}$ is a bounded and connected open subset $\Omega$ of $\mathbb{R}^{n}$, whose boundary $\partial \Omega$ is Lipschitz-continuous, the set $\Omega$ being locally on a single side of $\partial \Omega$.

Prepared using sagej.cls 
Let $\omega$ be a domain in $\mathbb{R}^{2}$, let $y=\left(y_{\alpha}\right)$ denote a generic point in $\omega$, and let $\partial_{\alpha}:=\partial / \partial y_{\alpha}$ and $\partial_{\alpha \beta}:=\partial^{2} / \partial y_{\alpha} \partial y_{\beta}$. A mapping $\boldsymbol{\theta} \in \mathcal{C}^{1}\left(\bar{\omega} ; \mathbb{E}^{3}\right)$ is an immersion if the two vectors

$$
\boldsymbol{a}_{\alpha}(y):=\partial_{\alpha} \boldsymbol{\theta}(y)
$$

are linearly independent at each point $y \in \bar{\omega}$. Then the image $\boldsymbol{\theta}(\bar{\omega})$ of the set $\bar{\omega}$ under the mapping $\boldsymbol{\theta}$ is a surface in $\mathbb{E}^{3}$, equipped with $y_{1}, y_{2}$ as its curvilinear coordinates. Given any point $y \in \bar{\omega}$, the vectors $\boldsymbol{a}_{\alpha}(y)$ span the tangent plane to the surface $\boldsymbol{\theta}(\bar{\omega})$ at the point $\boldsymbol{\theta}(y)$, the unit vector

$$
\boldsymbol{a}_{3}(y):=\frac{\boldsymbol{a}_{1}(y) \wedge \boldsymbol{a}_{2}(y)}{\left|\boldsymbol{a}_{1}(y) \wedge \boldsymbol{a}_{2}(y)\right|}
$$

is normal to $\boldsymbol{\theta}(\bar{\omega})$ at $\boldsymbol{\theta}(y)$, the three vectors $\boldsymbol{a}_{i}(y)$ form the covariant basis at $\boldsymbol{\theta}(y)$, and the three vectors $\boldsymbol{a}^{j}(y)$ defined by the relations

$$
\boldsymbol{a}^{j}(y) \cdot \boldsymbol{a}_{i}(y)=\delta_{i}^{j}
$$

form the contravariant basis at $\boldsymbol{\theta}(y)$; note that the vectors $\boldsymbol{a}^{\beta}(y)$ also span the tangent plane to $\boldsymbol{\theta}(\bar{\omega})$ at $\boldsymbol{\theta}(y)$ and that $\boldsymbol{a}^{3}(y)=\boldsymbol{a}_{3}(y)$.

The first fundamental form of the surface $\boldsymbol{\theta}(\bar{\omega})$ is defined by means of its covariant components

$$
a_{\alpha \beta}:=\boldsymbol{a}_{\alpha} \cdot \boldsymbol{a}_{\beta}=a_{\beta \alpha} \in \mathcal{C}^{0}(\bar{\omega}),
$$

or by means of its contravariant components

$$
a^{\alpha \beta}:=\boldsymbol{a}^{\alpha} \cdot \boldsymbol{a}^{\beta}=a^{\beta \alpha} \in \mathcal{C}^{0}(\bar{\omega}) .
$$

Note that the symmetric matrix field $\left(a^{\alpha \beta}\right)$ is the inverse of the matrix field $\left(a_{\alpha \beta}\right)$, that $\boldsymbol{a}^{\beta}=a^{\alpha \beta} \boldsymbol{a}_{\alpha}$ and $\boldsymbol{a}_{\alpha}=a_{\alpha \beta} \boldsymbol{a}^{\beta}$, and that the area element along $\boldsymbol{\theta}(\bar{\omega})$ is given at each point $\boldsymbol{\theta}(y), y \in \bar{\omega}$, by $\sqrt{a(y)} \mathrm{d} y$, where

$$
a:=\operatorname{det}\left(a_{\alpha \beta}\right) \in \mathcal{C}^{0}(\bar{\omega}) .
$$

Given an immersion $\boldsymbol{\theta} \in \mathcal{C}^{2}\left(\bar{\omega} ; \mathbb{E}^{3}\right)$, the second fundamental form of the surface $\boldsymbol{\theta}(\bar{\omega})$ is defined by means of its covariant components

$$
b_{\alpha \beta}:=\partial_{\alpha} \boldsymbol{a}_{\beta} \cdot \boldsymbol{a}_{3}=-\boldsymbol{a}_{\beta} \cdot \partial_{\alpha} \boldsymbol{a}_{3}=b_{\beta \alpha} \in \mathcal{C}^{0}(\bar{\omega}),
$$

or by means of its mixed components

$$
b_{\alpha}^{\beta}:=a^{\beta \sigma} b_{\alpha \sigma} \in \mathcal{C}^{0}(\bar{\omega})
$$

and the Christoffel symbols associated with the immersion $\boldsymbol{\theta}$ are defined by

$$
\Gamma_{\alpha \beta}^{\sigma}:=\partial_{\alpha} \boldsymbol{a}_{\beta} \cdot \boldsymbol{a}^{\sigma}=\Gamma_{\beta \alpha}^{\sigma} \in \mathcal{C}^{0}(\bar{\omega}) .
$$

The Gaussian curvature at each point $\boldsymbol{\theta}(y), y \in \bar{\omega}$, of the surface $\boldsymbol{\theta}(\bar{\omega})$ is defined by

$$
\kappa(y):=\frac{\operatorname{det}\left(b_{\alpha \beta}(y)\right)}{\operatorname{det}\left(a_{\alpha \beta}(y)\right)}=\operatorname{det}\left(b_{\alpha}^{\beta}(y)\right)
$$

Prepared using sagej.cls 
(the denominator in the above relation does not vanish since $\boldsymbol{\theta}$ is assumed to be an immersion). Note that the Gaussian curvature $\kappa(y)$ at the point $\boldsymbol{\theta}(y)$ is also equal to the product of the two principal curvatures at this point.

A surface $\boldsymbol{\theta}(\bar{\omega})$ defined by means of an immersion $\boldsymbol{\theta} \in \mathcal{C}^{2}\left(\bar{\omega} ; \mathbb{E}^{3}\right)$ is said to be elliptic if its Gaussian curvature is everywhere $>0$ in $\bar{\omega}$, or equivalently, if there exists a constant $\kappa_{0}$ such that

$$
0<\kappa_{0} \leq \kappa(y) \quad \text { for all } y \in \bar{\omega} .
$$

Given an immersion $\boldsymbol{\theta} \in \mathcal{C}^{2}\left(\bar{\omega} ; \mathbb{E}^{3}\right)$ and a vector field $\boldsymbol{\eta}=\left(\eta_{i}\right) \in \mathcal{C}^{1}\left(\bar{\omega} ; \mathbb{R}^{3}\right)$, the vector field

$$
\tilde{\boldsymbol{\eta}}:=\eta_{i} \boldsymbol{a}^{i}
$$

can be viewed as a displacement field of the surface $\boldsymbol{\theta}(\bar{\omega})$, thus defined by means of its covariant components $\eta_{i}$ over the vectors $\boldsymbol{a}^{i}$ of the contravariant bases along the surface. If the norms $\left\|\eta_{i}\right\|_{\mathcal{C}^{1}(\bar{\omega})}$ are small enough, the mapping $\left(\boldsymbol{\theta}+\eta_{i} \boldsymbol{a}^{i}\right) \in \mathcal{C}^{1}\left(\bar{\omega} ; \mathbb{E}^{3}\right)$ is also an immersion, so that the set $\left(\boldsymbol{\theta}+\eta_{i} \boldsymbol{a}^{i}\right)(\bar{\omega})$ is also a surface in $\mathbb{E}^{3}$, equipped with the same curvilinear coordinates as those of the surface $\boldsymbol{\theta}(\bar{\omega})$, called the deformed surface corresponding to the displacement field $\tilde{\boldsymbol{\eta}}=\eta_{i} \boldsymbol{a}^{i}$. One can then define the first fundamental form of the deformed surface by means of its covariant components

$$
a_{\alpha \beta}(\boldsymbol{\eta}):=\left(\boldsymbol{a}_{\alpha}+\partial_{\alpha} \tilde{\boldsymbol{\eta}}\right) \cdot\left(\boldsymbol{a}_{\beta}+\partial_{\beta} \tilde{\boldsymbol{\eta}}\right),
$$

and the second fundamental form of the deformed surface by means of its covariant components

$$
b_{\alpha \beta}(\boldsymbol{\eta}):=\partial_{\alpha}\left(\boldsymbol{a}_{\beta}+\partial_{\beta} \tilde{\boldsymbol{\eta}}\right) \cdot \frac{\left(\boldsymbol{a}_{1}+\partial_{1} \tilde{\boldsymbol{\eta}}\right) \wedge\left(\boldsymbol{a}_{2}+\partial_{2} \tilde{\boldsymbol{\eta}}\right)}{\left|\left(\boldsymbol{a}_{1}+\partial_{1} \tilde{\boldsymbol{\eta}}\right) \wedge\left(\boldsymbol{a}_{2}+\partial_{2} \tilde{\boldsymbol{\eta}}\right)\right|}
$$

The linear part with respect to $\tilde{\boldsymbol{\eta}}$ in the difference $\frac{1}{2}\left(a_{\alpha \beta}(\boldsymbol{\eta})-a_{\alpha \beta}\right)$ is called the linearized change of metric tensor associated with the displacement field $\eta_{i} \boldsymbol{a}^{i}$, the covariant components of which are then given by

$$
\begin{aligned}
\gamma_{\alpha \beta}(\boldsymbol{\eta}) & =\frac{1}{2}\left(\boldsymbol{a}_{\alpha} \cdot \partial_{\beta} \tilde{\boldsymbol{\eta}}+\partial_{\alpha} \tilde{\boldsymbol{\eta}} \cdot \boldsymbol{a}_{\beta}\right) \\
& =\frac{1}{2}\left(\partial_{\beta} \eta_{\alpha}+\partial_{\alpha} \eta_{\beta}\right)-\Gamma_{\alpha \beta}^{\sigma} \eta_{\sigma}-b_{\alpha \beta} \eta_{3}=\gamma_{\beta \alpha}(\boldsymbol{\eta}) .
\end{aligned}
$$

The linear part with respect to $\tilde{\boldsymbol{\eta}}$ in the difference $\left(b_{\alpha \beta}(\boldsymbol{\eta})-b_{\alpha \beta}\right)$ is called the linearized change of curvature tensor associated with the displacement field $\eta_{i} \boldsymbol{a}^{i}$, the covariant components of which are then given by

$$
\begin{aligned}
\rho_{\alpha \beta}(\boldsymbol{\eta}) & =\left(\partial_{\alpha \beta} \tilde{\boldsymbol{\eta}}-\Gamma_{\alpha \beta}^{\sigma} \partial_{\sigma} \tilde{\boldsymbol{\eta}}\right) \cdot \boldsymbol{a}_{3} \\
& =\partial_{\alpha \beta} \eta_{3}-\Gamma_{\alpha \beta}^{\sigma} \partial_{\sigma} \eta_{3}-b_{\alpha}^{\sigma} b_{\sigma \beta} \eta_{3} \\
& +b_{\alpha}^{\sigma}\left(\partial_{\beta} \eta_{\sigma}-\Gamma_{\beta \sigma}^{\tau} \eta_{\tau}\right)+b_{\beta}^{\tau}\left(\partial_{\alpha} \eta_{\tau}-\Gamma_{\alpha \tau}^{\sigma} \eta_{\sigma}\right) \\
& +\left(\partial_{\alpha} b_{\beta}^{\tau}+\Gamma_{\alpha \sigma}^{\tau} b_{\beta}^{\sigma}-\Gamma_{\alpha \beta}^{\sigma} b_{\sigma}^{\tau}\right) \eta_{\tau}=\rho_{\beta \alpha}(\boldsymbol{\eta}) .
\end{aligned}
$$

Let us now recall the definition of the time-dependent version of the linearised change of metric tensor $\gamma_{\alpha \beta}$. Consider the operator

$$
\tilde{\gamma}_{\alpha \beta}: L^{2}\left(0, T ; H^{1}(\omega) \times H^{1}(\omega) \times L^{2}(\omega)\right) \rightarrow L^{2}\left(0, T ; L^{2}(\omega)\right),
$$

Prepared using sagej.cls 
defined by

$$
\tilde{\gamma}_{\alpha \beta}(\boldsymbol{\eta})(t):=\gamma_{\alpha \beta}(\boldsymbol{\eta}(t)) \text { for all } \boldsymbol{\eta} \in L^{2}\left(0, T ; H^{1}(\omega) \times H^{1}(\omega) \times L^{2}(\omega)\right),
$$

for almost all (a.a. in what follows) $t \in(0, T)$. This operator is well-defined, linear, and continuous (cf., [24]).

Let us also recall the definition of the time-dependent version of the linearised change of curvature tensor $\rho_{\alpha \beta}$. Consider the operator

$$
\tilde{\rho}_{\alpha \beta}: L^{2}\left(0, T ; H^{1}(\omega) \times H^{1}(\omega) \times H^{2}(\omega)\right) \rightarrow L^{2}\left(0, T ; L^{2}(\omega)\right)
$$

defined by

$$
\tilde{\rho}_{\alpha \beta}(\boldsymbol{\eta})(t):=\rho_{\alpha \beta}(\boldsymbol{\eta}(t)) \text { for all } \boldsymbol{\eta} \in L^{2}\left(0, T ; H^{1}(\omega) \times H^{1}(\omega) \times H^{2}(\omega)\right),
$$

for a.a. $t \in(0, T)$. This operator is clearly well-defined, linear, and continuous (cf., [24]).

\section{A natural model for time-dependent flexural shells}

Let $\omega$ be a domain in $\mathbb{R}^{2}$ with boundary $\gamma$, and let $\gamma_{0}$ be a non-empty relatively open subset of $\gamma$. Let $I$ be an interval of the form $(0, T)$, with $T<\infty$.

For each $\varepsilon>0$, we define the sets

$$
\left.\Omega^{\varepsilon}:=\omega \times\right]-\varepsilon, \varepsilon\left[\text { and } \Gamma_{ \pm}^{\varepsilon}:=\omega \times\{ \pm \varepsilon\}\right.
$$

we let $x^{\varepsilon}=\left(x_{i}^{\varepsilon}\right)$ designate a generic point in the set $\overline{\Omega^{\varepsilon}}$, and let $\partial_{i}^{\varepsilon}:=\partial / \partial x_{i}^{\varepsilon}$. Hence we have $x_{\alpha}^{\varepsilon}=y_{\alpha}$ and $\partial_{\alpha}^{\varepsilon}=\partial_{\alpha}$. Define, also, the set

$$
\Gamma_{0}^{\varepsilon}:=\gamma_{0} \times[-\varepsilon, \varepsilon],
$$

which is thus a subset of the lateral face of the undeformed reference configuration.

In all that follows, we are given an injective immersion $\boldsymbol{\theta} \in \mathcal{C}^{3}\left(\bar{\omega} ; \mathbb{E}^{3}\right)$ and $\varepsilon>0$, and we consider a shell with middle surface $\boldsymbol{\theta}(\bar{\omega})$ and with constant thickness $2 \varepsilon$. This means that the reference configuration of the shell is the set $\Theta\left(\overline{\Omega^{\varepsilon}}\right)$, where the mapping $\Theta: \overline{\Omega^{\varepsilon}} \rightarrow \mathbb{E}^{3}$ is defined by

$$
\boldsymbol{\Theta}\left(x^{\varepsilon}\right):=\boldsymbol{\theta}(y)+x_{3}^{\varepsilon} \boldsymbol{a}^{3}(y) \text { at each point } x^{\varepsilon}=\left(y, x_{3}^{\varepsilon}\right) \in \overline{\Omega^{\varepsilon}} .
$$

Note that the injectivity assumption is made here for physical reasons, but that is otherwise not needed in the proofs. One can then show (cf. Theorem 3.1-1 of [21] or Theorem 4.1-1 of [22]) that, if the thickness $\varepsilon>0$ is small enough, such a mapping $\Theta \in \mathcal{C}^{2}\left(\overline{\Omega^{\varepsilon}} ; \mathbb{E}^{3}\right)$ is a $\mathcal{C}^{2}$-diffeomorphism from $\overline{\Omega^{\varepsilon}}$ onto $\Theta\left(\overline{\Omega^{\varepsilon}}\right)$, hence is in particular an injective immersion, in the sense that the three vectors

$$
\boldsymbol{g}_{i}^{\varepsilon}\left(x^{\varepsilon}\right):=\partial_{i}^{\varepsilon} \boldsymbol{\Theta}\left(x^{\varepsilon}\right)
$$

are linearly independent at each point $x^{\varepsilon} \in \overline{\Omega^{\varepsilon}}$; these vectors then constitute the covariant basis at the point $\boldsymbol{\Theta}\left(x^{\varepsilon}\right)$, while the three vectors $\boldsymbol{g}^{j, \varepsilon}\left(x^{\varepsilon}\right)$, defined by the relations

$$
\boldsymbol{g}^{j, \varepsilon}\left(x^{\varepsilon}\right) \cdot \boldsymbol{g}_{i}^{\varepsilon}\left(x^{\varepsilon}\right)=\delta_{i}^{j}
$$

constitute the contravariant basis at the same point.

It will be implicitly assumed in what follows that the immersion $\boldsymbol{\theta} \in \mathcal{C}^{3}\left(\bar{\omega} ; \mathbb{E}^{3}\right)$ is injective and that $\varepsilon>0$ is small enough so that $\Theta: \overline{\Omega^{\varepsilon}} \rightarrow \mathbb{E}^{3}$ is a $\mathcal{C}^{2}$-diffeomorphism onto its image.

We henceforth assume that the shell is made of a homogeneous and isotropic linearly elastic material and that its reference configuration $\Theta\left(\overline{\Omega^{\varepsilon}}\right)$ is a natural state, i.e., is stress free. As a result

Prepared using sagej.cls 
of these assumptions, the elastic behavior of this elastic material is completely characterized by its two Lamé constants $\lambda \geq 0$ and $\mu>0$ (see, e.g., Section 3.8 in 25). The positive constant $\rho$ designates the mass density of the shell per unit volume.

We also assume that the shell is subjected to applied body forces whose density per unit volume is defined by means of its contravariant components $f^{i, \varepsilon} \in L^{\infty}\left(0, T ; L^{2}\left(\Omega^{\varepsilon}\right)\right)$, i.e., over the vectors $\boldsymbol{g}_{i}^{\varepsilon}$ of the covariant bases; to applied surface forces whose density per unit area is defined by means of its contravariant components $h^{i, \varepsilon} \in L^{\infty}\left(0, T ; L^{2}\left(\Gamma_{+}^{\varepsilon} \cup \Gamma_{-}^{\varepsilon}\right)\right)$, i.e., over the vectors $\boldsymbol{g}_{i}^{\varepsilon}$ of the covariant bases; and to a homogeneous boundary condition of place along the portion $\Gamma_{0}^{\varepsilon}$ of its lateral face, i.e., the admissible displacement fields vanish on $\Gamma_{0}^{\varepsilon}$. For a.a. $t \in(0, T)$, we can thus define the contravariant components $p^{i, \varepsilon}(t)$ of the vector $\boldsymbol{p}^{\varepsilon}=\left(p^{i, \varepsilon}\right)$ over the vectors $\boldsymbol{a}_{i}$ of the covariant bases by

$$
p^{i, \varepsilon}(t):=\left\{\int_{-\varepsilon}^{\varepsilon} f^{i, \varepsilon}(t) \mathrm{d} x_{3}^{\varepsilon}+h_{+}^{i, \varepsilon}(t)+h_{-}^{i, \varepsilon}(t)\right\} \in L^{2}(\omega) \text { for a.a. } t \in(0, T),
$$

where $h_{ \pm}^{i, \varepsilon}(t):=h^{i, \varepsilon}(t)(\cdot, \pm \varepsilon) \in L^{2}(\omega)$, for a.a. $t \in(0, T)$.

Define the space

$$
\boldsymbol{V}_{K}(\omega):=\left\{\boldsymbol{\eta}=\left(\eta_{i}\right) \in H^{1}(\omega) \times H^{1}(\omega) \times H^{2}(\omega) ; \eta_{i}=\partial_{\nu} \eta_{3}=0 \text { on } \gamma_{0}\right\},
$$

where the symbol $\partial_{\nu}$ denotes the outer unit normal derivative operator along $\gamma$. The space $\boldsymbol{V}_{K}(\omega)$ is the one used for formulating the two-dimensional equations governing Koiter's model (see the series of papers [26, [11, [18] and [17]).

Define the norm $\|\cdot\|_{\boldsymbol{V}_{K}(\omega)}$ by

$$
\|\boldsymbol{\eta}\|_{\boldsymbol{V}_{K}(\omega)}:=\left\{\sum_{\alpha}\left\|\eta_{\alpha}\right\|_{1, \omega}^{2}+\left\|\eta_{3}\right\|_{2, \omega}^{2}\right\}^{1 / 2} \quad \text { for each } \boldsymbol{\eta}=\left(\eta_{i}\right) \in \boldsymbol{V}_{K}(\omega),
$$

Next, we define the fourth-order two-dimensional elasticity tensor of the shell, viewed here as a two-dimensional linearly elastic body, by means of its contravariant components

$$
a^{\alpha \beta \sigma \tau}:=\frac{4 \lambda \mu}{\lambda+2 \mu} a^{\alpha \beta} a^{\sigma \tau}+2 \mu\left(a^{\alpha \sigma} a^{\beta \tau}+a^{\alpha \tau} a^{\beta \sigma}\right) .
$$

Following the terminology proposed in Section 6.1 of [21, a linearly elastic shell is said to be a flexural shell if the following two additional assumptions are satisfied: first, length $\gamma_{0}>0$ (an assumption that is satisfied if $\gamma_{0}$ is a non-empty relatively open subset of $\gamma$, as here), and second, the following space of admissible linearized inextensional displacements:

$$
\begin{aligned}
\boldsymbol{V}_{F}(\omega):=\left\{\boldsymbol{\eta}=\left(\eta_{i}\right) \in H^{1}(\omega) \times H^{1}(\omega) \times H^{2}(\omega) ;\right. \\
\left.\eta_{i}=\partial_{\nu} \eta_{3}=0 \text { on } \gamma_{0} \text { and } \gamma_{\alpha \beta}(\boldsymbol{\eta})=0 \text { in } \omega\right\},
\end{aligned}
$$

contains nonzero functions, i.e.,

$$
\boldsymbol{V}_{F}(\omega) \neq\{\mathbf{0}\} .
$$

Classical examples of flexural shells are, for instance, cylindrical shells, conical shells and plates (see, respectively, Figures 6.1-1, 6.1-2, and 6.1-3 of [21]).

To begin with, we state a crucial inequality that holds for general surfaces. 
Theorem 3.1. Let $\omega$ be a domain in $\mathbb{R}^{2}$ and let $\boldsymbol{\theta} \in \mathcal{C}^{3}\left(\bar{\omega} ; \mathbb{E}^{3}\right)$ be an immersion. Let $\gamma_{0}$ be a non-empty relatively open subset of $\gamma$. Define the space

$$
\boldsymbol{V}_{K}(\omega):=\left\{\boldsymbol{\eta}=\left(\eta_{i}\right) \in H^{1}(\omega) \times H^{1}(\omega) \times H^{2}(\omega) ; \eta_{i}=\partial_{\nu} \eta_{3}=0 \text { on } \gamma_{0}\right\} .
$$

Then there exists a constant $c=c\left(\omega, \gamma_{0}, \boldsymbol{\theta}\right)>0$ such that

$$
\left\{\sum_{\alpha}\left\|\eta_{\alpha}\right\|_{1, \omega}^{2}+\left\|\eta_{3}\right\|_{2, \omega}^{2}\right\}^{1 / 2} \leq c\left\{\sum_{\alpha, \beta}\left\|\gamma_{\alpha \beta}(\boldsymbol{\eta})\right\|_{0, \omega}^{2}+\sum_{\alpha, \beta}\left\|\rho_{\alpha \beta}(\boldsymbol{\eta})\right\|_{0, \omega}^{2}\right\}^{1 / 2}
$$

for all $\boldsymbol{\eta}=\left(\eta_{i}\right) \in \boldsymbol{V}_{K}(\omega)$.

The above inequality, which is due to Bernadou \& Ciarlet [27] and was later improved by Bernadou, Ciarlet \& Miara [28] (see also Theorem 2.6-4 of [21]), constitutes an example of a Korn inequality on a general surface; it constitutes a "Korn inequality" in the sense that it provides a basic estimate of an appropriate norm of a displacement field defined on a surface in terms of an appropriate norm of a specific "measure of strain" (here, the linearized change of metric tensor and the linearized change of curvature tensor) corresponding to the displacement field under consideration.

A natural formulation of a set of time-dependent two-dimensional equations ("two-dimensional", in the sense that they are posed over the two-dimensional subset $\omega$ ) can be derived by slightly modifying the model proposed by Xiao in the paper [20, where time-dependent Koiter's shells are studied.

Let us introduce the problem $\mathcal{P}_{F}^{\varepsilon}(\omega)$, which constitutes the point of departure of our analysis.

Problem $\mathcal{P}_{F}^{\varepsilon}(\omega)$. Find a vector field $\boldsymbol{\zeta}^{\varepsilon}=\left(\zeta_{i}^{\varepsilon}\right):(0, T) \rightarrow \boldsymbol{V}_{F}(\omega)$ such that

$$
\begin{aligned}
& \boldsymbol{\zeta}^{\varepsilon} \in L^{\infty}\left(0, T ; \boldsymbol{V}_{F}(\omega)\right), \\
& \dot{\boldsymbol{\zeta}}^{\varepsilon} \in L^{\infty}\left(0, T ; \boldsymbol{L}^{2}(\omega)\right), \\
& \ddot{\boldsymbol{\zeta}}^{\varepsilon} \in L^{\infty}\left(0, T ; \boldsymbol{V}_{F}^{*}(\omega)\right),
\end{aligned}
$$

that satisfies the following variational equations

$$
2 \varepsilon^{3} \rho \frac{\mathrm{d}^{2}}{\mathrm{~d} t^{2}} \int_{\omega} \zeta_{i}^{\varepsilon}(t) \eta_{i} \sqrt{a} \mathrm{~d} y+\frac{\varepsilon^{3}}{3} \int_{\omega} a^{\alpha \beta \sigma \tau} \rho_{\sigma \tau}\left(\boldsymbol{\zeta}^{\varepsilon}(t)\right) \rho_{\alpha \beta}(\boldsymbol{\eta}) \sqrt{a} \mathrm{~d} y=\int_{\omega} p^{i, \varepsilon}(t) \eta_{i} \sqrt{a} \mathrm{~d} y,
$$

for all $\boldsymbol{\eta}=\left(\eta_{i}\right) \in \boldsymbol{V}_{F}(\omega)$, in the sense of distributions in $(0, T)$, and that satisfies the following initial conditions

$$
\left\{\begin{array}{l}
\boldsymbol{\zeta}^{\varepsilon}(0)=\boldsymbol{\zeta}_{0} \\
\dot{\zeta}^{\varepsilon}(0)=\boldsymbol{\zeta}_{1}
\end{array}\right.
$$

where $\boldsymbol{\zeta}_{0} \in \boldsymbol{V}_{F}(\omega)$ and $\boldsymbol{\zeta}_{1} \in \boldsymbol{L}^{2}(\omega)$ are prescribed.

We say that $\boldsymbol{\zeta}^{\varepsilon}$ is a weak solution of Problem $\mathcal{P}_{F}^{\varepsilon}(\omega)$ if

$$
\begin{aligned}
& \boldsymbol{\zeta}^{\varepsilon} \in L^{\infty}\left(0, T ; \boldsymbol{V}_{F}(\omega)\right), \\
& \dot{\boldsymbol{\zeta}}^{\varepsilon} \in L^{\infty}\left(0, T ; \boldsymbol{L}^{2}(\omega)\right), \\
& \ddot{\boldsymbol{\zeta}}^{\varepsilon} \in L^{\infty}\left(0, T ; \boldsymbol{V}_{F}^{*}(\omega)\right),
\end{aligned}
$$

Prepared using sagej.cls 
if $\boldsymbol{\zeta}^{\varepsilon}$ satisfies the variational equations of Problem $\mathcal{P}_{F}^{\varepsilon}(\omega)$ in the sense of distributions in $(0, T)$, and also satisfies the initial conditions (1).

We say that $\boldsymbol{\zeta}^{\varepsilon}$ is a strong solution of Problem $\mathcal{P}_{F}^{\varepsilon}(\omega)$ if

$$
\boldsymbol{\zeta}^{\varepsilon} \in \mathcal{C}^{0}\left([0, T] ; \boldsymbol{V}_{F}(\omega)\right) \cap \mathcal{C}^{1}\left([0, T] ; \boldsymbol{L}^{2}(\omega)\right),
$$

if $\boldsymbol{\zeta}^{\varepsilon}$ satisfies the variational equations of Problem $\mathcal{P}_{F}^{\varepsilon}(\omega)$ in the sense of distributions in $(0, T)$, and also satisfies the initial conditions (1). Let us recall that the existence and uniqueness of a strong solution to Problem $\mathcal{P}_{F}^{\varepsilon}(\omega)$ has been established in Theorem 4.1 of 24. The proof was carried out by generalizing a classical argument, which can be found in [29].

\section{Penalisation of the considered problem}

To fix the ideas, from now onward, we identify $L^{2}(\omega)$ and $\boldsymbol{L}^{2}(\omega)$ with their respective dual spaces, and we equip them with the following inner products

$$
\begin{gathered}
(\eta, \xi) \in L^{2}(\omega) \times L^{2}(\omega) \rightarrow \int_{\omega} \eta \xi \sqrt{a} \mathrm{~d} y, \\
(\boldsymbol{\eta}, \boldsymbol{\xi}) \in \boldsymbol{L}^{2}(\omega) \times \boldsymbol{L}^{2}(\omega) \rightarrow \int_{\omega} \eta_{i} \xi_{i} \sqrt{a} \mathrm{~d} y .
\end{gathered}
$$

It is worth mentioning that these inner products are also conventionally denoted by the symbols $(\cdot, \cdot)_{L^{2}(\omega)}$ and $(\cdot, \cdot)_{L^{2}(\omega)}$, respectively.

A possible way to prove the existence and uniqueness of solutions of Problem $\mathcal{P}_{F}^{\varepsilon}(\omega)$ without relying on the abstract functional spaces introduced in Section 4 of [24] (see also 29]), consists in adapting the penalty scheme described in Chapter II, Section 4 of [30] (see also [31]) to formulate an alternate problem posed over the function space $\boldsymbol{V}_{K}(\omega)$, which does not take into account the constraint appearing in the definition of the space $\boldsymbol{V}_{F}(\omega)$.

Observe first that $\boldsymbol{V}_{K}(\omega)$ is dense in $\boldsymbol{L}^{2}(\omega)$ and that

$$
\boldsymbol{V}_{K}(\omega) \hookrightarrow \hookrightarrow \boldsymbol{L}^{2}(\omega) \hookrightarrow \hookrightarrow \boldsymbol{V}_{K}^{*}(\omega) .
$$

Let $\kappa>0$ denote the penalty parameter and let us introduce the corresponding "penalised" problem $\mathcal{P}_{F, \kappa}^{\varepsilon}(\omega)$.

Problem $\mathcal{P}_{F, \kappa}^{\varepsilon}(\omega)$. Find a vector field $\boldsymbol{\zeta}_{\kappa}^{\varepsilon}=\left(\zeta_{i, \kappa}^{\varepsilon}\right):[0, T] \rightarrow \boldsymbol{V}_{K}(\omega)$ such that

$$
\boldsymbol{\zeta}_{\kappa}^{\varepsilon} \in \mathcal{C}^{0}\left([0, T] ; \boldsymbol{V}_{K}(\omega)\right) \cap \mathcal{C}^{1}\left([0, T] ; \boldsymbol{L}^{2}(\omega)\right),
$$

that satisfies the following variational equations

$$
\begin{gathered}
2 \varepsilon^{3} \rho \frac{\mathrm{d}^{2}}{\mathrm{~d} t^{2}} \int_{\omega} \zeta_{i, \kappa}^{\varepsilon}(t) \eta_{i} \sqrt{a} \mathrm{~d} y+\frac{\varepsilon^{3}}{3} \int_{\omega} a^{\alpha \beta \sigma \tau} \rho_{\sigma \tau}\left(\boldsymbol{\zeta}_{\kappa}^{\varepsilon}(t)\right) \rho_{\alpha \beta}(\boldsymbol{\eta}) \sqrt{a} \mathrm{~d} y \\
+\frac{1}{\kappa} \int_{\omega} a^{\alpha \beta \sigma \tau} \gamma_{\sigma \tau}\left(\boldsymbol{\zeta}_{\kappa}^{\varepsilon}(t)\right) \gamma_{\alpha \beta}(\boldsymbol{\eta}) \sqrt{a} \mathrm{~d} y=\int_{\omega} p^{i, \varepsilon}(t) \eta_{i} \sqrt{a} \mathrm{~d} y
\end{gathered}
$$

for all $\boldsymbol{\eta} \in \boldsymbol{V}_{K}(\omega)$, in the sense of distributions in $(0, T)$, and which satisfies the initial conditions (1). 
We say that $\boldsymbol{\zeta}_{\kappa}^{\varepsilon}$ is a weak solution of Problem $\mathcal{P}_{F, \kappa}^{\varepsilon}(\omega)$ if

$$
\begin{aligned}
& \boldsymbol{\zeta}_{\kappa}^{\varepsilon} \in L^{\infty}\left(0, T ; \boldsymbol{V}_{K}(\omega)\right), \\
& \dot{\boldsymbol{\zeta}}_{\kappa}^{\varepsilon} \in L^{\infty}\left(0, T ; \boldsymbol{L}^{2}(\omega)\right), \\
& \ddot{\boldsymbol{\zeta}}_{\kappa}^{\varepsilon} \in L^{\infty}\left(0, T ; \boldsymbol{V}_{K}^{*}(\omega)\right),
\end{aligned}
$$

if $\boldsymbol{\zeta}_{\kappa}^{\varepsilon}$ satisfies the variational equations of Problem $\mathcal{P}_{F, \kappa}^{\varepsilon}(\omega)$ in the sense of distributions in $(0, T)$, and also satisfies the initial conditions (1).

We say that $\boldsymbol{\zeta}_{\kappa}^{\varepsilon}$ is a strong solution of Problem $\mathcal{P}_{F, \kappa}^{\varepsilon}(\omega)$ if

$$
\boldsymbol{\zeta}_{\kappa}^{\varepsilon} \in \mathcal{C}^{0}\left([0, T] ; \boldsymbol{V}_{K}(\omega)\right) \cap \mathcal{C}^{1}\left([0, T] ; \boldsymbol{L}^{2}(\omega)\right),
$$

if $\boldsymbol{\zeta}_{\kappa}^{\varepsilon}$ satisfies the variational equations of Problem $\mathcal{P}_{F, \kappa}^{\varepsilon}(\omega)$ in the sense of distributions in $(0, T)$, and also satisfies the initial conditions (1).

For each $\kappa>0$, let us define the bilinear form $a_{\kappa}: \boldsymbol{V}_{K}(\omega) \times \boldsymbol{V}_{K}(\omega) \rightarrow \mathbb{R}$ by

$$
a_{\kappa}(\boldsymbol{\xi}, \boldsymbol{\eta}):=\frac{\varepsilon^{3}}{3} \int_{\omega} a^{\alpha \beta \sigma \tau} \rho_{\sigma \tau}(\boldsymbol{\xi}) \rho_{\alpha \beta}(\boldsymbol{\eta}) \sqrt{a} \mathrm{~d} y+\frac{1}{\kappa} \int_{\omega} a^{\alpha \beta \sigma \tau} \gamma_{\sigma \tau}(\boldsymbol{\xi}) \gamma_{\alpha \beta}(\boldsymbol{\eta}) \sqrt{a} \mathrm{~d} y
$$

The bilinear form $a_{\kappa}(\cdot, \cdot)$ is continuous over the space $\boldsymbol{V}_{K}(\omega)$, i.e., there exists a constant $C_{\kappa}>0$, which depends on $\kappa$, such that

$$
\left|a_{\kappa}(\boldsymbol{\xi}, \boldsymbol{\eta})\right| \leq C_{\kappa}\|\boldsymbol{\xi}\|_{\boldsymbol{V}_{K}(\omega)}\|\boldsymbol{\eta}\|_{\boldsymbol{V}_{K}(\omega)}, \quad \text { for all } \boldsymbol{\xi}, \boldsymbol{\eta} \in \boldsymbol{V}_{K}(\omega)
$$

For $\kappa>0$ sufficiently small (recall that the small parameter $\varepsilon>0$ is fixed), the uniform positivedefiniteness of the elasticity tensor of the shell $\left(a^{\alpha \beta \sigma \tau}\right)$ (cf. Theorem 3.3-2 of [21]) and Korn's inequality on a general surface (Theorem 3.1) give the existence of a constant $c>0$ such that

$$
\begin{aligned}
a_{\kappa}(\boldsymbol{\eta}, \boldsymbol{\eta}) & =\frac{\varepsilon^{3}}{3} \int_{\omega} a^{\alpha \beta \sigma \tau} \rho_{\sigma \tau}(\boldsymbol{\eta}) \rho_{\alpha \beta}(\boldsymbol{\eta}) \sqrt{a} \mathrm{~d} y+\frac{1}{\kappa} \int_{\omega} a^{\alpha \beta \sigma \tau} \gamma_{\sigma \tau}(\boldsymbol{\eta}) \gamma_{\alpha \beta}(\boldsymbol{\eta}) \sqrt{a} \mathrm{~d} y \\
& \geq \frac{\varepsilon^{3}}{3} \sum_{\alpha, \beta}\left\{\left\|\rho_{\alpha \beta}(\boldsymbol{\eta})\right\|_{0, \omega}^{2}+\left\|\gamma_{\alpha \beta}(\boldsymbol{\eta})\right\|_{0, \omega}^{2}\right\} \\
& \geq c\left\{\sum_{\alpha}\left\|\eta_{\alpha}\right\|_{1, \omega}^{2}+\left\|\eta_{3}\right\|_{2, \omega}^{2}\right\}
\end{aligned}
$$

for all $\boldsymbol{\eta} \in \boldsymbol{V}_{K}(\omega)$, namely, the bilinear form $a_{\kappa}(\cdot, \cdot)$ is $\boldsymbol{V}_{K}(\omega)$-elliptic.

Let us recall that Problem $\mathcal{P}_{F, \kappa}^{\varepsilon}(\omega)$ admits a unique strong solution (see, e.g., Theorem 5.1 of [24]).

Theorem 4.1. Problem $\mathcal{P}_{F, \kappa}^{\varepsilon}(\omega)$ admits a unique strong solution $\boldsymbol{\zeta}_{\kappa}^{\varepsilon} \in \mathcal{C}^{0}\left([0, T] ; \boldsymbol{V}_{K}(\omega)\right) \cap$ $\mathcal{C}^{1}\left([0, T] ; \boldsymbol{L}^{2}(\omega)\right)$.

Letting $\kappa \rightarrow 0$, we obtain that the family $\left(\boldsymbol{\zeta}_{\kappa}^{\varepsilon}\right)_{\kappa>0}$ of solutions to Problem $\mathcal{P}_{F, \kappa}^{\varepsilon}(\omega)$ converges to the unique strong solution $\boldsymbol{\zeta}^{\varepsilon}$ of Problem $\mathcal{P}_{F}^{\varepsilon}(\omega)$ with the following modes of convergences 
(cf., Theorem 6.1 of [24]):

$$
\begin{array}{ll}
\boldsymbol{\zeta}_{\kappa}^{\varepsilon} \stackrel{*}{\rightarrow} \boldsymbol{\zeta}^{\varepsilon}, & \text { in } L^{\infty}\left(0, T ; \boldsymbol{V}_{K}(\omega)\right) \text { as } \kappa \rightarrow 0, \\
\dot{\boldsymbol{\zeta}}_{\kappa}^{\varepsilon} \stackrel{*}{*}^{\varepsilon}, & \text { in } L^{\infty}\left(0, T ; \boldsymbol{L}^{2}(\omega)\right) \text { as } \kappa \rightarrow 0, \\
\ddot{\boldsymbol{\zeta}}_{\kappa}^{\varepsilon} \stackrel{*}{*}^{\varepsilon}, & \text { in } L^{\infty}\left(0, T ; \boldsymbol{V}_{F}^{*}(\omega)\right) \text { as } \kappa \rightarrow 0, \\
\boldsymbol{\zeta}_{\kappa}^{\varepsilon} \rightarrow \boldsymbol{\zeta}^{\varepsilon}, & \text { in } \mathcal{C}^{0}\left([0, T] ; \boldsymbol{L}^{2}(\omega)\right) \text { as } \kappa \rightarrow 0, \\
\dot{\boldsymbol{\zeta}}_{\kappa}^{\varepsilon} \rightarrow \dot{\boldsymbol{\zeta}}^{\varepsilon}, & \text { in } \mathcal{C}^{0}\left([0, T] ; \boldsymbol{V}_{F}^{*}(\omega)\right) \text { as } \kappa \rightarrow 0 .
\end{array}
$$

The main existence and uniqueness result can be then rigorously stated.

Theorem 4.2. Problem $\mathcal{P}_{F}^{\varepsilon}(\omega)$ admits a unique weak solution $\boldsymbol{\zeta}^{\varepsilon}$.

\section{Semi-discretisation in space by means of a conforming finite element method}

In this section we rigorously present a suitable finite element method to approximate the solution of Problem $\mathcal{P}_{F, \kappa}^{\varepsilon}(\omega)$. Following [32] and [33, we recall some basic terminology and definitions. In what follows the letter $h$ denotes a quantity approaching zero. We denote by $\left(\mathcal{T}_{h}\right)_{h>0}$ a family of triangulations of the domain $\omega$, henceforth assumed to be polygonal, made of triangles and we let $T$ denote any element of such a family. Let us first recall, following [32] and [33], the rigorous definition of finite element in $\mathbb{R}^{n}$, where $n \geq 1$ is an integer.

A finite element in $\mathbb{R}^{n}$ is a triple $(T, P, \mathcal{N})$ where:

(i) $T$ is a closed subset of $\mathbb{R}^{n}$ with a nonempty interior and a Lipschitz-continuous boundary,

(ii) $P$ is a finite dimensional space of real-valued functions defined over $T$,

(iii) $\mathcal{N}$ is is a finite set of linearly independent linear forms $N_{i}, 1 \leq i \leq \operatorname{dim} P$, defined over the space $P$.

By definition, it is assumed that the set $\mathcal{N}$ is $P$-unisolvent in the following sense: given any real scalars $\alpha_{i}, 1 \leq i \leq \operatorname{dim} P$, there exists a unique function $g \in P$ which satisfies

$$
N_{i}(g)=\alpha_{i}, \quad 1 \leq i \leq \operatorname{dim} P .
$$

It is worth mentioning that each well-defined finite element satisfies the following requirement (cf., e.g., page 79 of [32]):

$$
\operatorname{dim} P=\operatorname{card} \mathcal{N} .
$$

It is henceforth assumed that the degrees of freedom, $N_{i}$, lie in the dual space of a function space larger than $P$ like, for instance, a Sobolev space (see [33]). For brevity we shall conform our terminology to the one of [32, calling the sole set $T$ a finite element. Define the diameter of any finite element $T$ as follows:

$$
h_{T}=\operatorname{diam} T:=\max _{x, y \in T}|x-y|
$$

Let us also define

$$
\rho_{T}:=\sup \{\operatorname{diam} B ; B \text { is a ball contained in } T\} .
$$

A triangulation $\mathcal{T}_{h}$ is said to be regular (cf., e.g., [32]) if:

Prepared using sagej.cls 
(i) There exists a constant $\sigma>0$, independent of $h$, such that

$$
\text { for all } T \in \mathcal{T}_{h}, \quad \frac{h_{T}}{\rho_{T}} \leq \sigma .
$$

(ii) The quantity $h:=\max \left\{h_{T}>0 ; T \in \mathcal{T}_{h}\right\}$ approaches zero.

There is of course an ambiguity in the meaning of $h$, which was first regarded as a parameter associated with the considered family of triangulations, and which next denotes a geometrical entity. Nevertheless, in this paper, we have conformed to this standard notation (cf., e.g., [32]). In the rest of this section, the parameter $h$ is assumed to be fixed.

The spatial variable is discretised by means of finite element method presented in 32 . We use a triangle of type (1) for approximating the tangential components $\zeta_{\alpha, \kappa}^{\varepsilon}$ of the sought displacement vector field, and a conforming $\mathcal{C}^{1}$ finite element, more specifically, a Hsieh-CloughTocher triangle (henceforth, $\mathrm{HCT}$ triangle), for approximating the transverse component $\zeta_{3, \kappa}^{\varepsilon}$ of the sought displacement vector field.

In what follows, the notation $P_{k}(K), k \geq 1$, designates the space formed by the restriction to a triangle $K \in \mathcal{T}_{h}$ of all the polynomials of degree $\leq k$ in two variables.

Let us now rigorously introduce the finite element spaces that will be exploited to numerically approximate the solution to the model under consideration. Define

$$
\boldsymbol{V}_{h}:=V_{h 1} \times V_{h 2} \times V_{h 3},
$$

where

$$
\begin{aligned}
V_{h \alpha}:= & \left\{\eta_{h} \in \mathcal{C}^{0}(\bar{\omega}) ;\left.\eta_{h}\right|_{K} \in P_{1}(K)\right. \text { for each } \\
& \left.K \in \mathcal{T}_{h} \text { and } \eta_{h}=0 \text { on } \gamma_{0}\right\},
\end{aligned}
$$

and

$$
\begin{aligned}
V_{h 3}:=\{ & \eta_{h} \in \mathcal{C}^{1}(\bar{\omega}) ;\left.\eta_{h}\right|_{K_{i}} \in P_{3}\left(K_{i}\right) \text { for all } 1 \leq i \leq 3 \\
& \text { and for all } K=\cup K_{i} \in \mathcal{T}_{h}, \\
& \eta_{h} \text { is } \mathcal{C}^{1}-\text { continuous at each interior vertex, } \\
& \partial_{\nu} \eta_{h} \text { is continuous at each mid-point of the interior edge, } \\
& \eta_{h}=0 \text { at each vertex that belongs to } \gamma_{0}, \\
& \left.\partial_{\nu} \eta_{h}=0 \text { at each mid-point that belongs to } \gamma_{0}\right\} .
\end{aligned}
$$

Let us introduce the semi-discrete approximate problem corresponding to Problem $\mathcal{P}_{F, \kappa}^{\varepsilon}(\omega)$, which is denoted in what follows by $\mathcal{P}_{F, h}^{\kappa}$. We drop the dependence on the parameter $\varepsilon$ for keeping the notation easy. It should however be observed that the solution to $\mathcal{P}_{F, h}^{\kappa}$ also depends on $\varepsilon$.

Problem $\mathcal{P}_{F, h}^{\kappa}$. Find a vector field $\boldsymbol{\zeta}_{h}^{\kappa}=\left(\zeta_{i, h}^{\kappa}\right):[0, T] \rightarrow \boldsymbol{V}_{h}$ that satisfies the following system of ordinary differential equations with respect to the variable $t$

$$
\begin{gathered}
2 \varepsilon^{3} \rho \frac{\mathrm{d}^{2}}{\mathrm{~d} t^{2}} \int_{\omega} \zeta_{i, h}^{\kappa}(t) \eta_{i, h} \sqrt{a} \mathrm{~d} y+\frac{\varepsilon^{3}}{3} \int_{\omega} a^{\alpha \beta \sigma \tau} \rho_{\sigma \tau}\left(\boldsymbol{\zeta}_{h}^{\kappa}(t)\right) \rho_{\alpha \beta}\left(\boldsymbol{\eta}_{h}\right) \sqrt{a} \mathrm{~d} y \\
+\frac{1}{\kappa} \int_{\omega} a^{\alpha \beta \sigma \tau} \gamma_{\sigma \tau}\left(\boldsymbol{\zeta}_{h}^{\kappa}(t)\right) \gamma_{\alpha \beta}\left(\boldsymbol{\eta}_{h}\right) \sqrt{a} \mathrm{~d} y=\int_{\omega} p^{i, \varepsilon}(t) \eta_{i, h} \sqrt{a} \mathrm{~d} y
\end{gathered}
$$

Prepared using sagej.cls 
for all $\boldsymbol{\eta}_{h} \in \boldsymbol{V}_{h}$ and which satisfies the initial conditions

$$
\left\{\begin{array}{l}
\boldsymbol{\zeta}_{h}^{\kappa}(0)=\boldsymbol{\zeta}_{0, h}, \\
\dot{\boldsymbol{\zeta}}_{h}^{\kappa}(0)=\boldsymbol{\zeta}_{1, h},
\end{array}\right.
$$

where $\boldsymbol{\zeta}_{0, h}$ is the projection of the given vector field $\boldsymbol{\zeta}_{0}$ onto the space $\boldsymbol{V}_{h}$ with respect to the standard inner product of $\boldsymbol{V}_{K}(\omega)$, while $\boldsymbol{\zeta}_{1, h}$ is the projection of the given vector field $\boldsymbol{\zeta}_{1}$ onto the space $\boldsymbol{V}_{h}$ with respect to the $\boldsymbol{L}^{2}(\omega)$ inner product (cf., e.g., page 13 of [24]).

We then have that the following existence and uniqueness result holds (cf. Theorem 8.4-1 of [34] or Section 14.5 of [35]).

Theorem 5.1. Problem $\mathcal{P}_{F, h}^{\kappa}$ admits a unique solution.

\section{Total discretisation in time}

The total discretisation in time of Problem $\overline{\mathcal{P}_{F, h}^{\kappa}}$ is performed using Newmark's scheme for hyperbolic equations (cf., e.g., the seminal paper [36] and Chapter 8 of [34]).

Let us consider a division of the interval $[0, T]$ made of $N$ points, where $N$ is any positive integer. Define the time step $\Delta t:=T / N$, the time instant $t_{n}:=n \Delta t$, the approximations $\zeta_{h}^{n, \kappa} \simeq \zeta_{h}^{\kappa}\left(t_{n}\right)$, and the vectors $\boldsymbol{p}^{n}:=\boldsymbol{p}^{\varepsilon}\left(t_{n}\right) \in \boldsymbol{L}^{2}(\omega)$, where $n=0, \ldots, N$.

We can then exhibit Newmark's scheme corresponding to Problem $\mathcal{P}_{F, h}^{\kappa}$

Algorithm . For each $n=0, \ldots, N-2$, find $\boldsymbol{\zeta}_{h}^{n} \in \boldsymbol{V}_{h}$ such that

$$
\begin{aligned}
& \frac{2 \varepsilon^{3} \rho}{(\Delta t)^{2}}\left(\boldsymbol{\zeta}_{h}^{n+2, \kappa}-2 \boldsymbol{\zeta}_{h}^{n+1, \kappa}+\boldsymbol{\zeta}_{h}^{n, \kappa}, \boldsymbol{e}_{h}\right)_{\boldsymbol{L}^{2}(\omega)} \\
& \quad+a_{\kappa}\left(\beta \boldsymbol{\zeta}_{h}^{n+2, \kappa}+\left(\frac{1}{2}-2 \beta+\gamma\right) \boldsymbol{\zeta}_{h}^{n+1, \kappa}+\left(\frac{1}{2}+\beta-\gamma\right) \boldsymbol{\zeta}_{h}^{n, \kappa}, \boldsymbol{e}_{h}\right) \\
& =\left(\beta \boldsymbol{p}^{n+2}+\left(\frac{1}{2}-2 \beta+\gamma\right) \boldsymbol{p}^{n+1}+\left(\frac{1}{2}+\beta-\gamma\right) \boldsymbol{p}^{n}, \boldsymbol{e}_{h}\right)_{\boldsymbol{L}^{2}(\omega)},
\end{aligned}
$$

for all $e_{h} \in V_{h}$.

where $\beta$ and $\gamma$ are given nonnegative real constants (cf., e.g., Section 8.5 of [34]).

The vector field $\boldsymbol{\zeta}_{h}^{1, \kappa}$ is obtained as the unique solution of the following variational equations

$$
\begin{aligned}
& \frac{2 \varepsilon^{3} \rho}{(\Delta t)^{2}}\left(\boldsymbol{\zeta}_{h}^{1, \kappa}-\boldsymbol{\zeta}_{0, h}-\Delta t \boldsymbol{\zeta}_{1, h}, \boldsymbol{e}_{h}\right)_{\boldsymbol{L}^{2}(\omega)} \\
& \quad+a_{\kappa}\left(\beta \boldsymbol{\zeta}_{h}^{1, \kappa}+\left(\frac{1}{2}-\beta\right) \boldsymbol{\zeta}_{0, h}, \boldsymbol{e}_{h}\right) \\
& =\left(\beta \boldsymbol{p}^{1}+\left(\frac{1}{2}-\beta\right) \boldsymbol{p}^{0}, \boldsymbol{e}_{h}\right)_{\boldsymbol{L}^{2}(\omega)}, \quad \text { for all } \boldsymbol{e}_{h} \in \boldsymbol{V}_{h} .
\end{aligned}
$$

Observe that the constants $\beta$ and $\gamma$ cannot be chosen arbitrarily. Indeed, following Section 8.6 of [34, the stability of Newmark's scheme is achieved when

$$
\gamma>\frac{1}{2} \quad \text { and } \quad \beta \geq \frac{(1 / 2+\gamma)^{2}}{4}
$$

Prepared using sagej.cls 
We recall that if

$$
\beta<\frac{(1 / 2+\gamma)^{2}}{4}
$$

then the validity of extra specific stability conditions must be verified (cf., e.g, Theorems 8.5-1 and $8.6-1$ of [34]).

Let us now discuss the convergence of the proposed algorithm in the case where the constants $\beta$ and $\gamma$ are chosen as in (6), the stability condition (8.6-30) in Theorem 8.6-2 of [34] holds, and the solution to Problem $\mathcal{P}_{F, \kappa}^{\varepsilon}(\omega)$ is more regular with respect to the variable $t$, i.e.,

$$
\boldsymbol{\zeta}_{\kappa}^{\varepsilon} \in \mathcal{C}^{2}\left([0, T] ; \boldsymbol{V}_{K}(\omega)\right) \cap \mathcal{C}^{3}\left([0, T] ; \boldsymbol{L}^{2}(\omega)\right) .
$$

Under these additional assumptions, for each $n=0, \ldots, N$, we obtain the following estimate:

$$
\left\|\boldsymbol{\zeta}_{h}^{n, \kappa}-\boldsymbol{\zeta}^{\varepsilon}\left(t_{n}\right)\right\|_{\boldsymbol{L}^{2}(\omega)} \leq\left\|\boldsymbol{\zeta}_{h}^{n, \kappa}-\boldsymbol{\zeta}_{\kappa}^{\varepsilon}\left(t_{n}\right)\right\|_{\boldsymbol{L}^{2}(\omega)}+\left\|\boldsymbol{\zeta}_{\kappa}^{\varepsilon}\left(t_{n}\right)-\boldsymbol{\zeta}^{\varepsilon}\left(t_{n}\right)\right\|_{\boldsymbol{L}^{2}(\omega)}=O(h)+O(\kappa) .
$$

This shows that, if $h$ and $\kappa$ are sufficiently small, the each approximate solution $\boldsymbol{\zeta}_{h}^{n, \kappa}$ output by (4)- (5), with $n=0, \ldots, N$, is a good approximation (in the sense of the $\boldsymbol{L}^{2}(\omega)$ norm) of the solution $\boldsymbol{\zeta}^{\varepsilon}$ of Problem $\mathcal{P}_{F}^{\varepsilon}(\omega)$ evaluated at the time instant $t_{n}$, again with $n=0, \ldots, N$. We also observe that, under additional regularity assumptions, the latter estimate can be improved (cf., e.g., Theorem 8.7-2 of [34]).

\section{Numerical experiments: Cylindrical shells}

We conduct our first set of numerical tests in the case where the middle surface of the flexural shell under consideration is a portion of a cylinder (cf. Figure 1 ).

We consider a domain $\omega$ shaped as follows

$$
\omega:=\left\{\left(y_{1}, y_{2}\right) \in \mathbb{R}^{2} ; 0<y_{1}<\pi \text { and } 0<y_{2}<1\right\}
$$

where $\gamma_{0}$, the region of the boundary at which the clamping occurs, takes the following form

$$
\gamma_{0}:=\left\{\left(y_{1}, y_{2}\right) \in \mathbb{R}^{2} ; y_{1}=\pi, y_{2} \in[0,1]\right\}
$$

In curvilinear coordinates, the middle surface is given by the mapping $\boldsymbol{\theta}$ defined by

$$
\boldsymbol{\theta}\left(y_{1}, y_{2}\right):=\left(r \cos y_{1}, r \sin y_{1}, h y_{2}\right), \quad \text { for all }\left(y_{1}, y_{2}\right) \in \bar{\omega}
$$

where $r>0$ and $h>0$.

Prepared using sagej.cls 


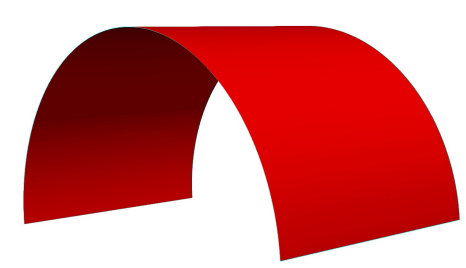

Figure 1. A cylindrical shell.

This means that the displacement field vanishes along one generatrix of the corresponding cylindrical surface. As initial conditions in Problem $\mathcal{P}_{F}^{\varepsilon}(\omega)$, we take $\boldsymbol{\zeta}_{0}=\boldsymbol{\zeta}_{1}=\mathbf{0}$.

Then the covariant basis of the tangent plane to $\boldsymbol{\theta}(\bar{\omega})$ at the point $\boldsymbol{\theta}\left(y_{1}, y_{2}\right)$ is given by

$$
\begin{aligned}
& \boldsymbol{a}_{1}=\left(-r \sin y_{1}, r \cos y_{1}, 0\right), \\
& \boldsymbol{a}_{2}=(0,0, h), \\
& \boldsymbol{a}_{3}=\boldsymbol{a}^{3}=\left(\cos y_{1}, \sin y_{1}, 0\right) .
\end{aligned}
$$

The covariant components of the first fundamental form of $\boldsymbol{\theta}(\bar{\omega})$ are given by

$$
a_{11}=r^{2}, \quad a_{12}=a_{21}=0, \quad a_{22}=h^{2},
$$

and

$$
a=\operatorname{det}\left(a_{\alpha \beta}\right)=r^{2} h^{2} .
$$

The contravariant components of metric tensors on $\boldsymbol{\theta}(\bar{\omega})$ are given by

$$
a^{11}=r^{-2}, \quad a^{12}=a^{21}=0, \quad a^{22}=h^{-2},
$$

so that we obtain the following

$$
\boldsymbol{a}^{1}=a^{11} \boldsymbol{a}_{1}=\left(-r^{-1} \sin y_{1}, r^{-1} \cos y_{1}, 0\right), \quad \boldsymbol{a}^{2}=a^{22} \boldsymbol{a}_{2}=\left(0,0, h^{-1}\right) .
$$

Since

$$
\begin{aligned}
\partial_{1} \boldsymbol{a}_{1} & =\left(-r \cos y_{1},-r \sin y_{1}, 0\right), \\
\partial_{2} \boldsymbol{a}_{1} & =(0,0,0), \\
\partial_{1} \boldsymbol{a}_{2}=\partial_{2} \boldsymbol{a}_{2} & =(0,0,0),
\end{aligned}
$$

then, the covariant and mixed components of the second fundamental form of $\boldsymbol{\theta}(\bar{\omega})$ are given by

$$
\begin{aligned}
b_{11} & =-r, \\
b_{12} & =b_{21}=b_{22}=0, \\
b_{1}^{1} & =a^{11} b_{11}=-r^{-1}, \\
b_{2}^{1} & =b_{1}^{2}=b_{2}^{2}=0 .
\end{aligned}
$$

Prepared using sagej.cls 
The Christoffel symbols of $\boldsymbol{\theta}(\bar{\omega})$ are given by

$$
\begin{aligned}
& \Gamma_{11}^{1}=\Gamma_{12}^{1}=\Gamma_{21}^{1}=\Gamma_{22}^{1}=0, \\
& \Gamma_{11}^{2}=\Gamma_{12}^{2}=\Gamma_{21}^{2}=\Gamma_{22}^{2}=0 .
\end{aligned}
$$

In what follows, we take $r=0.20 \mathrm{~m}, h=0.40 \mathrm{~m}$ (cf. [37]). We take the Young's modulus as in [37], i.e.,

$$
E=2.1 \times 10^{11} \mathrm{~Pa},
$$

and the Poisson ratio as (cf. [37])

$$
\nu=0.3
$$

Since the Lamé constants, the Young's modulus (7) and the Poisson's ratio (8) are related as follows

$$
\lambda=\frac{E \nu}{(1+\nu)(1-2 \nu)}, \quad \mu=\frac{E}{2(1+\nu)},
$$

so that, plugging (7) and (8) into (9), we obtain

$$
\lambda=1.21 \times 10^{11} \mathrm{~Pa}, \quad \mu=8.08 \times 10^{10} \mathrm{~Pa} .
$$

The mass density per unit volume of the shell is taken as in [37, i.e.,

$$
\rho=7.85 \times 10^{3} \mathrm{~kg} / \mathrm{m}^{3} .
$$

For the time being, we set the penalty parameter $\kappa$ equal to $10^{-6}$ and $\gamma=0.6$.

To recapitulate, the parameters used in the experiments that output Figure 3 are the following

$$
\begin{aligned}
r & =0.20 \mathrm{~m}, \\
h & =0.40 \mathrm{~m}, \\
\varepsilon & =0.002 \mathrm{~m}, \\
E & =2.1 \times 10^{11} \mathrm{~Pa}, \\
\nu & =0.3, \\
\rho & =7.85 \times 10^{3} \mathrm{~kg} / \mathrm{m}^{3}, \\
\kappa & =10^{-6}, \\
\gamma & =0.6, \\
\beta & =\frac{(1 / 2+\gamma)^{2}}{4} .
\end{aligned}
$$

We implement Newmark's scheme (4)- (5) by means of the software FreeFem++ (see [38]), and we visualise the results using ParaView (see [39]).

In our experiments we use a mesh made of 2,700 elements, as displayed in Figure 2. 


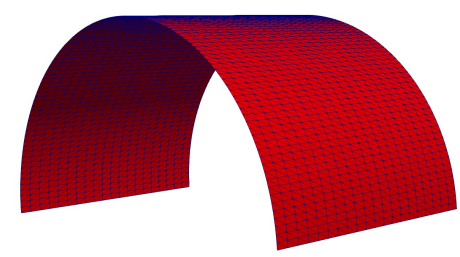

Figure 2. Mesh of the cylindrical surface $\boldsymbol{\theta}(\bar{\omega})$ made of 2,700 elements.

We consider the following dynamic loads

$$
\begin{aligned}
& p^{1, \varepsilon}\left(t, y_{1}, y_{2}\right)=0 \\
& p^{2, \varepsilon}\left(t, y_{1}, y_{2}\right)=0 \\
& p^{3, \varepsilon}\left(t, y_{1}, y_{2}\right)=20 t y_{1}
\end{aligned}
$$

The deformations of the cylindrical shell under consideration at different time instants are shown in Figure 3 for a time-step $\Delta t=0.01 \mathrm{~s}$. The colouring meaning is described by the vertical gauge on the right hand side of each mesh. The generatrix on the left part of the displayed cylinders corresponds to the clamped part of the boundary. In the following implementation, we applied a scaling factor of order 1,000 in ParaView, to visualise a more progressive evolution of the displacement field magnitude.

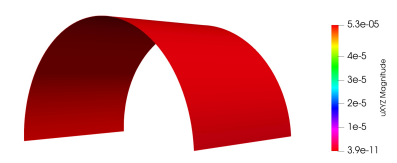

(a) $t=0.00 \mathrm{~s}$

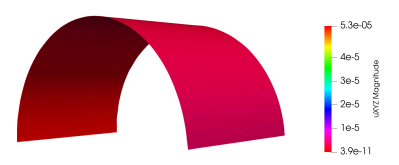

(b) $t=0.10 \mathrm{~s}$

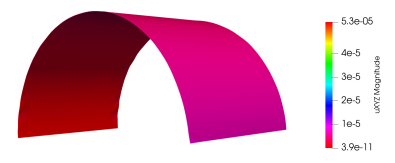

(c) $t=0.20 \mathrm{~s}$

Prepared using sagej.cls 

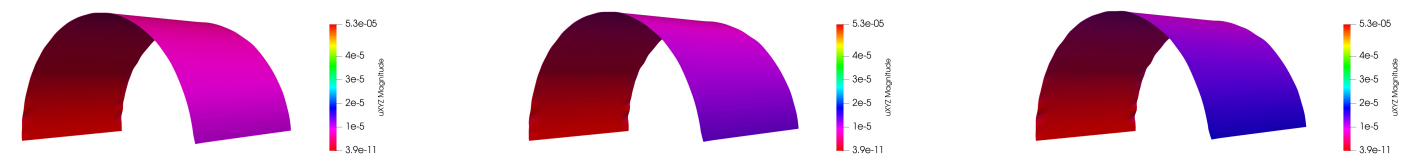

(d) $t=0.30 \mathrm{~s}$

(e) $t=0.40 \mathrm{~s}$

(f) $t=0.50 \mathrm{~s}$
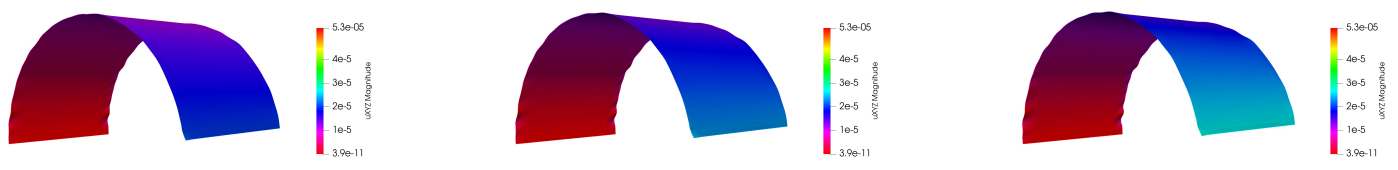

(g) $t=0.60 \mathrm{~s}$

(h) $t=0.70 \mathrm{~s}$

(i) $t=0.80 \mathrm{~s}$
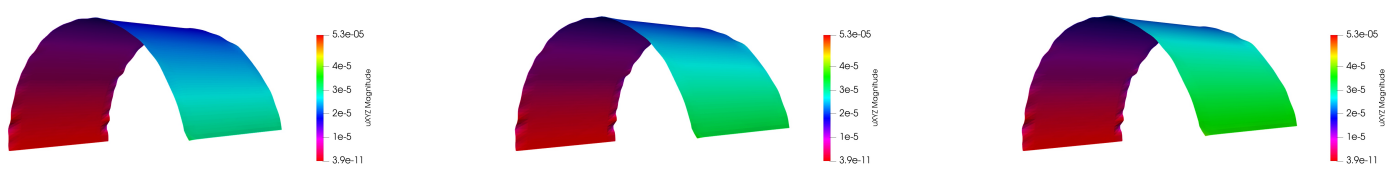

\section{(j) $t=0.90 \mathrm{~s}$}

(k) $t=1.00 \mathrm{~s}$

(I) $t=1.10 \mathrm{~s}$

Figure 3. $\mathrm{n}=90, \mathrm{~m}=30, \Delta t=0.01$. The number of points of the mesh that are processed by the software FreeFem ++ is determined by the quantities $n$ and $m$.

In the following tables 1, 2, and 3, we illustrate the deformation of the middle surface of the shell, by displaying how the displacement fields at three random points of the middle surface of the shell change as time passes, and the penalty parameter $\kappa$ varies.

Prepared using sagej.cls 
Table 1. Displacement vectors at three random points of the middle surface of the shell at time instants $t=0.01 \mathrm{~s}$ and $t=0.05 \mathrm{~s}$, with time-step $\Delta t=0.01$, and penalty parameters $\kappa=10^{-6}$ and $\kappa=10^{-8}$.

\begin{tabular}{c|cc|c|cc}
\hline \hline $\begin{array}{c}\text { Penalty } \\
\text { parameter }\end{array}$ & \multicolumn{2}{|c|}{$\kappa=10^{-6}$} & $\begin{array}{c}\text { Penalty } \\
\text { parameter }\end{array}$ & \multicolumn{2}{c}{$\kappa=10^{-8}$} \\
\hline Time & $t=0.01 \mathrm{~s}$ & $t=0.05 \mathrm{~s}$ & Time & $t=0.01 \mathrm{~s}$ & $t=0.05 \mathrm{~s}$ \\
\hline $\mathrm{x}$ & $-1.30384 \mathrm{e}-06$ & $-3.04204 \mathrm{e}-06$ & $\mathrm{x}$ & $4.12591 \mathrm{e}-09$ & $9.63102 \mathrm{e}-09$ \\
$\mathrm{y}$ & $-8.39842 \mathrm{e}-09$ & $-1.95938 \mathrm{e}-08$ & $\mathrm{y}$ & $8.92493 \mathrm{e}-09$ & $2.08305 \mathrm{e}-08$ \\
$\mathrm{z}$ & $4.51353 \mathrm{e}-52$ & $1.78963 \mathrm{e}-51$ & $\mathrm{z}$ & $-1.08597 \mathrm{e}-54$ & $-6.8079 \mathrm{e}-54$ \\
\hline $\mathrm{x}$ & $-5.26531 \mathrm{e}-07$ & $-1.22844 \mathrm{e}-06$ & $\mathrm{x}$ & $-4.56679 \mathrm{e}-10$ & $-1.06501 \mathrm{e}-09$ \\
$\mathrm{y}$ & $-3.2931 \mathrm{e}-07$ & $-7.68309 \mathrm{e}-07$ & $\mathrm{y}$ & $7.66398 \mathrm{e}-09$ & $1.78874 \mathrm{e}-08$ \\
$\mathrm{z}$ & $1.37419 \mathrm{e}-40$ & $4.04022 \mathrm{e}-40$ & $\mathrm{z}$ & $7.2741 \mathrm{e}-44$ & $4.59639 \mathrm{e}-43$ \\
\hline $\mathrm{x}$ & $2.91321 \mathrm{e}-07$ & $6.79693 \mathrm{e}-07$ & $\mathrm{x}$ & $-1.82165 \mathrm{e}-09$ & $-4.25175 \mathrm{e}-09$ \\
$\mathrm{y}$ & $-1.63053 \mathrm{e}-07$ & $-3.80427 \mathrm{e}-07$ & $\mathrm{y}$ & $7.19426 \mathrm{e}-10$ & $1.67921 \mathrm{e}-09$ \\
$\mathrm{z}$ & $-4.21245 \mathrm{e}-28$ & $2.74884 \mathrm{e}-28$ & $\mathrm{z}$ & $-8.03791 \mathrm{e}-32$ & $1.79875 \mathrm{e}-31$ \\
\hline
\end{tabular}

Table 2. Displacement vectors at three random points of the middle surface of the shell at time instants $t=0.60 \mathrm{~s}$ and $t=0.90 \mathrm{~s}$, with time-step $\Delta t=0.01$, and penalty parameters $\kappa=10^{-8}$ and $\kappa=10^{-12}$.

\begin{tabular}{c|cc|c|cc}
\hline \hline $\begin{array}{c}\text { Penalty } \\
\text { parameter }\end{array}$ & \multicolumn{2}{|c|}{$\kappa=10^{-8}$} & $\begin{array}{c}\text { Penalty } \\
\text { parameter }\end{array}$ & \multicolumn{2}{c}{$\kappa=10^{-12}$} \\
\hline Time & $t=0.60 \mathrm{~s}$ & $t=0.90 \mathrm{~s}$ & Time & $t=0.60 \mathrm{~s}$ & $t=0.90 \mathrm{~s}$ \\
\hline $\mathrm{x}$ & $8.54092 \mathrm{e}-08$ & $1.26722 \mathrm{e}-07$ & $\mathrm{x}$ & $-2.70342 \mathrm{e}-11$ & $-4.01393 \mathrm{e}-11$ \\
$\mathrm{y}$ & $1.84684 \mathrm{e}-07$ & $2.73988 \mathrm{e}-07$ & $\mathrm{y}$ & $-1.73792 \mathrm{e}-13$ & $-2.5811 \mathrm{e}-13$ \\
$\mathrm{z}$ & $-3.65128 \mathrm{e}-51$ & $-1.19752 \mathrm{e}-50$ & $\mathrm{z}$ & $-3.75327 \mathrm{e}-54$ & $-8.87914 \mathrm{e}-54$ \\
\hline $\mathrm{x}$ & $-9.42638 \mathrm{e}-09$ & $-1.39806 \mathrm{e}-08$ & $\mathrm{x}$ & $-1.0906 \mathrm{e}-11$ & $-1.61949 \mathrm{e}-11$ \\
$\mathrm{y}$ & $1.58591 \mathrm{e}-07$ & $2.35278 \mathrm{e}-07$ & $\mathrm{y}$ & $-6.82084 \mathrm{e}-12$ & $-1.01286 \mathrm{e}-11$ \\
$\mathrm{z}$ & $2.48864 \mathrm{e}-40$ & $8.12662 \mathrm{e}-40$ & $\mathrm{z}$ & $4.69783 \mathrm{e}-43$ & $1.09072 \mathrm{e}-42$ \\
\hline $\mathrm{x}$ & $-3.7694 \mathrm{e}-08$ & $-5.59281 \mathrm{e}-08$ & $\mathrm{x}$ & $6.03201 \mathrm{e}-12$ & $8.95624 \mathrm{e}-12$ \\
$\mathrm{y}$ & $1.48882 \mathrm{e}-08$ & $2.20905 \mathrm{e}-08$ & $\mathrm{y}$ & $-3.37652 \mathrm{e}-12$ & $-5.0134 \mathrm{e}-12$ \\
$\mathrm{z}$ & $3.21975 \mathrm{e}-28$ & $7.90172 \mathrm{e}-28$ & $\mathrm{z}$ & $1.09084 \mathrm{e}-30$ & $1.49654 \mathrm{e}-30$ \\
\hline
\end{tabular}

Table 3. Displacement vectors at three random points of the middle surface of the shell at time instants $t=1.00 \mathrm{~s}$ and $t=1.20 \mathrm{~s}$, with time-step $\Delta t=0.01$, and penalty parameters $\kappa=10^{-6}$ and $\kappa=10^{-12}$.

\begin{tabular}{c|cc|c|cc}
\hline $\begin{array}{c}\text { Penalty } \\
\text { parameter }\end{array}$ & \multicolumn{2}{|c|}{$\kappa=10^{-6}$} & $\begin{array}{c}\text { Penalty } \\
\text { parameter }\end{array}$ & \multicolumn{2}{c}{$\kappa=10^{-12}$} \\
\hline Time & $t=1.00 \mathrm{~s}$ & $t=1.20 \mathrm{~s}$ & Time & $t=1.00 \mathrm{~s}$ & $t=1.20 \mathrm{~s}$ \\
\hline $\mathrm{x}$ & $-4.43328 \mathrm{e}-05$ & $-5.3025 \mathrm{e}-05$ & $\mathrm{x}$ & $-4.44892 \mathrm{e}-11$ & $-5.31926 \mathrm{e}-11$ \\
$\mathrm{y}$ & $-2.85605 \mathrm{e}-07$ & $-3.41629 \mathrm{e}-07$ & $\mathrm{y}$ & $-2.86123 \mathrm{e}-13$ & $-3.42136 \mathrm{e}-13$ \\
$\mathrm{z}$ & $3.45195 \mathrm{e}-48$ & $5.81452 \mathrm{e}-48$ & $\mathrm{z}$ & $-1.23685 \mathrm{e}-53$ & $-2.09736 \mathrm{e}-53$ \\
\hline $\mathrm{x}$ & $-1.7904 \mathrm{e}-05$ & $-2.14152 \mathrm{e}-05$ & $\mathrm{x}$ & $-1.79514 \mathrm{e}-11$ & $-2.1465 \mathrm{e}-11$ \\
$\mathrm{y}$ & $-1.11978 \mathrm{e}-05$ & $-1.33938 \mathrm{e}-05$ & $\mathrm{y}$ & $-1.12272 \mathrm{e}-11$ & $-1.34246 \mathrm{e}-11$ \\
$\mathrm{z}$ & $1.18632 \mathrm{e}-36$ & $2.10533 \mathrm{e}-36$ & $\mathrm{z}$ & $1.51811 \mathrm{e}-42$ & $2.56202 \mathrm{e}-42$ \\
\hline $\mathrm{x}$ & $9.90621 \mathrm{e}-06$ & $1.18531 \mathrm{e}-05$ & $\mathrm{x}$ & $9.92774 \mathrm{e}-12$ & $1.1872 \mathrm{e}-11$ \\
$\mathrm{y}$ & $-5.5445 \mathrm{e}-06$ & $-6.63435 \mathrm{e}-06$ & $\mathrm{y}$ & $-5.55716 \mathrm{e}-12$ & $-6.6454 \mathrm{e}-12$ \\
$\mathrm{z}$ & $-1.79321 \mathrm{e}-24$ & $-1.58878 \mathrm{e}-24$ & $\mathrm{z}$ & $1.62687 \mathrm{e}-30$ & $2.02813 \mathrm{e}-30$ \\
\hline \hline
\end{tabular}

The following tables 4,6 , and 8 report the evaluations of the norms $\left\|\boldsymbol{\zeta}_{h}^{n, \kappa}-\boldsymbol{\zeta}_{h}^{n, \kappa^{\prime}}\right\|_{\boldsymbol{L}^{2}(\omega)}$, i.e., the $\boldsymbol{L}^{2}(\omega)$-residuals of the approximate solutions evaluated at the same time instant $t_{n}$, constructed using the same mesh size $h$, but corresponding to different values $\kappa$ and $\kappa^{\prime}$ of the penalty parameter.

The purpose of tables 4, 6, and 8] is to numerically confirm that for all time instants $t_{n}$, the sequence $\left(\zeta_{h}^{n, \kappa}\right)_{\kappa>0}$ is a Cauchy sequence in $\boldsymbol{L}^{2}(\omega)$. The data we obtained agree with the conclusion 
of Theorems 4.1 and 4.2 In particular, we recall, again, that in the proof of the latter (cf. 24]), the following convergence was obtained (cf. (2)):

$$
\boldsymbol{\zeta}_{\kappa}^{\varepsilon} \rightarrow \boldsymbol{\zeta}^{\varepsilon}, \quad \text { in } \mathcal{C}^{0}\left([0, T] ; \boldsymbol{L}^{2}(\omega)\right) \text { as } \kappa \rightarrow 0
$$

Tables 5. 7, and 9 confirm that the same conclusion holds if the value of $\gamma$ changes, provided, however, that $\gamma \geq 1 / 2$.

Table 4. Let $\gamma=0.6$. The following table reports the measurements of the $\boldsymbol{L}^{2}(\omega)$-residuals of the approximate solutions at a given time instant corresponding to $\kappa=10^{-6}$ and $\kappa^{\prime}=10^{-12}$, respectively.

\begin{tabular}{cc}
\hline \hline Time & $\kappa=10^{-6}$ and $\kappa^{\prime}=10^{-12}$ \\
\hline $0.10 \mathrm{~s}$ & $9.105035061047419 \mathrm{e}-09$ \\
$0.20 \mathrm{~s}$ & $3.058470544333727 \mathrm{e}-08$ \\
$0.30 \mathrm{~s}$ & $6.449066960406247 \mathrm{e}-08$ \\
$0.40 \mathrm{~s}$ & $1.1132909131539029 \mathrm{e}-07$ \\
$0.50 \mathrm{~s}$ & $1.709097468250036 \mathrm{e}-07$ \\
$0.60 \mathrm{~s}$ & $2.4282536616866065 \mathrm{e}-07$ \\
$0.70 \mathrm{~s}$ & $3.268063050746276 \mathrm{e}-07$ \\
$0.80 \mathrm{~s}$ & $4.235323308277976 \mathrm{e}-07$ \\
$0.90 \mathrm{~s}$ & $5.340243748167916 \mathrm{e}-07$ \\
$1.00 \mathrm{~s}$ & $6.566224369986849 \mathrm{e}-07$ \\
$1.10 \mathrm{~s}$ & $7.938369859511519 \mathrm{e}-07$ \\
$1.20 \mathrm{~s}$ & $9.393890067106388 \mathrm{e}-07$ \\
$1.30 \mathrm{~s}$ & $1.0992038539893267 \mathrm{e}-06$ \\
$1.40 \mathrm{~s}$ & $1.2735584835066714 \mathrm{e}-06$ \\
\hline
\end{tabular}

Table 5. Let $\gamma=0.8$. The following table reports the measurements of the $\boldsymbol{L}^{2}(\omega)$-residuals of the approximate solutions at a given time instant, corresponding to $\kappa=10^{-6}$ and $\kappa^{\prime}=10^{-12}$, respectively.

\begin{tabular}{cc}
\hline \hline Time & $\kappa=10^{-6}$ and $\kappa^{\prime}=10^{-12}$ \\
\hline $0.10 \mathrm{~s}$ & $9.13022435949277 \mathrm{e}-09$ \\
$0.20 \mathrm{~s}$ & $3.070720172772241 \mathrm{e}-08$ \\
$0.30 \mathrm{~s}$ & $6.494699395353533 \mathrm{e}-08$ \\
$0.40 \mathrm{~s}$ & $1.118847259753477 \mathrm{e}-07$ \\
$0.50 \mathrm{~s}$ & $1.7143718680912363 \mathrm{e}-07$ \\
$0.60 \mathrm{~s}$ & $2.4377342081430797 \mathrm{e}-07$ \\
$0.70 \mathrm{~s}$ & $3.289644619106159 \mathrm{e}-07$ \\
$0.80 \mathrm{~s}$ & $4.2650850161955456 \mathrm{e}-07$ \\
$0.90 \mathrm{~s}$ & $5.367803325526587 \mathrm{e}-07$ \\
$1.00 \mathrm{~s}$ & $6.599655611907805 \mathrm{e}-07$ \\
$1.10 \mathrm{~s}$ & $7.958275201187722 \mathrm{e}-07$ \\
$1.20 \mathrm{~s}$ & $9.432864826616698 \mathrm{e}-07$ \\
$1.30 \mathrm{~s}$ & $1.1050444278868415 \mathrm{e}-06$ \\
$1.40 \mathrm{~s}$ & $1.279027281245845 \mathrm{e}-06$ \\
\hline
\end{tabular}


Table 6. Let $\gamma=0.6$. The following table reports the measurements of the $\boldsymbol{L}^{2}(\omega)$-residuals of the approximate solutions at a given time instant, corresponding to $\kappa=10^{-8}$ and $\kappa^{\prime}=10^{-12}$, respectively.

\begin{tabular}{cc}
\hline \hline Time & $\kappa=10^{-8}$ and $\kappa^{\prime}=10^{-12}$ \\
\hline $0.10 \mathrm{~s}$ & $9.092922670431935 \mathrm{e}-13$ \\
$0.20 \mathrm{~s}$ & $3.0551785666233502 \mathrm{e}-12$ \\
$0.30 \mathrm{~s}$ & $6.469394300280324 \mathrm{e}-12$ \\
$0.40 \mathrm{~s}$ & $1.1151143158076022 \mathrm{e}-11$ \\
$0.50 \mathrm{~s}$ & $1.7097534957261284 \mathrm{e}-11$ \\
$0.60 \mathrm{~s}$ & $2.4308028522236183 \mathrm{e}-11$ \\
$0.70 \mathrm{~s}$ & $3.2755013818368175 \mathrm{e}-11$ \\
$0.80 \mathrm{~s}$ & $4.2462762579475536 \mathrm{e}-11$ \\
$0.90 \mathrm{~s}$ & $5.351133923228302 \mathrm{e}-11$ \\
$1.00 \mathrm{~s}$ & $6.576490708244588 \mathrm{e}-11$ \\
$0.10 \mathrm{~s}$ & $7.913665593178143 \mathrm{e}-11$ \\
$1.20 \mathrm{~s}$ & $9.40539128322055 \mathrm{e}-11$ \\
$1.30 \mathrm{~s}$ & $1.101622383552461 \mathrm{e}-10$ \\
$1.40 \mathrm{~s}$ & $1.2757241762698354 \mathrm{e}-10$ \\
\hline \hline
\end{tabular}

Table 8. Let $\gamma=0.6$. The following table reports the measurements of the $\boldsymbol{L}^{2}(\omega)$-residuals of the approximate solutions at a given time instant, corresponding to $\kappa=10^{-6}$ and $\kappa^{\prime}=10^{-8}$, respectively.

\begin{tabular}{cc}
\hline \hline Time & $\kappa=10^{-6}$ and $\kappa^{\prime}=10^{-8}$ \\
\hline $0.10 \mathrm{~s}$ & $8.923964821442561 \mathrm{e}-09$ \\
$0.20 \mathrm{~s}$ & $2.9976395853899877 \mathrm{e}-08$ \\
$0.30 \mathrm{~s}$ & $6.320529449051073 \mathrm{e}-08$ \\
$0.40 \mathrm{~s}$ & $1.0911183801720238 \mathrm{e}-07$ \\
$0.50 \mathrm{~s}$ & $1.6750799347387358 \mathrm{e}-07$ \\
$0.60 \mathrm{~s}$ & $2.3799061869290796 \mathrm{e}-07$ \\
$0.70 \mathrm{~s}$ & $3.2029549991047165 \mathrm{e}-07$ \\
$0.80 \mathrm{~s}$ & $4.150932011593057 \mathrm{e}-07$ \\
$0.90 \mathrm{~s}$ & $5.233865140892298 \mathrm{e}-07$ \\
$1.00 \mathrm{~s}$ & $6.43545490928386 \mathrm{e}-07$ \\
$1.10 \mathrm{~s}$ & $7.780641080033482 \mathrm{e}-07$ \\
$1.20 \mathrm{~s}$ & $9.20683782879014 \mathrm{e}-07$ \\
$1.30 \mathrm{~s}$ & $1.0773057672601421 \mathrm{e}-06$ \\
$1.40 \mathrm{~s}$ & $1.248193238692263 \mathrm{e}-06$ \\
\hline \hline
\end{tabular}

Table 7. Let $\gamma=0.8$. The following table reports the measurements of the $\boldsymbol{L}^{2}(\omega)$-residuals of the approximate solutions at a given time instant corresponding to $\kappa=10^{-8}$ and $\kappa^{\prime}=10^{-12}$, respectively.

\begin{tabular}{cc}
\hline \hline Time & $\kappa=10^{-8}$ and $\kappa^{\prime}=10^{-12}$ \\
\hline $0.10 \mathrm{~s}$ & $9.094450770700801 \mathrm{e}-13$ \\
$0.20 \mathrm{~s}$ & $3.0567125182168985 \mathrm{e}-12$ \\
$0.30 \mathrm{~s}$ & $6.472054232239331 \mathrm{e}-12$ \\
$0.40 \mathrm{~s}$ & $1.1146741953492408 \mathrm{e}-11$ \\
$0.50 \mathrm{~s}$ & $1.707449421183145 \mathrm{e}-11$ \\
$0.60 \mathrm{~s}$ & $2.429095630126807 \mathrm{e}-11$ \\
$0.70 \mathrm{~s}$ & $3.273958834829574 \mathrm{e}-11$ \\
$0.80 \mathrm{~s}$ & $4.248234062530869 \mathrm{e}-11$ \\
$0.90 \mathrm{~s}$ & $5.3447325375494504 \mathrm{e}-11$ \\
$1.00 \mathrm{~s}$ & $6.571991607584821 \mathrm{e}-11$ \\
$1.10 \mathrm{~s}$ & $7.924678756800441 \mathrm{e}-11$ \\
$1.20 \mathrm{~s}$ & $9.405025523912434 \mathrm{e}-11$ \\
$1.30 \mathrm{~s}$ & $1.1008702063087494 \mathrm{e}-10$ \\
$1.40 \mathrm{~s}$ & $1.2746396794594706 \mathrm{e}-10$ \\
\hline \hline
\end{tabular}

Table 9. Let $\gamma=0.8$. The following table reports the measurements of the $\boldsymbol{L}^{2}(\omega)$-residuals of the approximate solutions at a given time instant, corresponding to $\kappa=10^{-6}$ and $\kappa^{\prime}=10^{-8}$, respectively.

\begin{tabular}{cc}
\hline Time step & $\kappa=10^{-6}$ and $\kappa^{\prime}=10^{-8}$ \\
\hline $0.10 \mathrm{~s}$ & $8.948887406047657 \mathrm{e}-09$ \\
$0.20 \mathrm{~s}$ & $3.0097516796473584 \mathrm{e}-08$ \\
$0.30 \mathrm{~s}$ & $6.365679263476454 \mathrm{e}-08$ \\
$0.40 \mathrm{~s}$ & $1.0966235518986009 \mathrm{e}-07$ \\
$0.50 \mathrm{~s}$ & $1.6803244705417505 \mathrm{e}-07$ \\
$0.60 \mathrm{~s}$ & $2.3893088971871273 \mathrm{e}-07$ \\
$0.70 \mathrm{~s}$ & $3.224336170677912 \mathrm{e}-07$ \\
$0.80 \mathrm{~s}$ & $4.1803768169472007 \mathrm{e}-07$ \\
$0.90 \mathrm{~s}$ & $5.261212689644947 \mathrm{e}-07$ \\
$1.00 \mathrm{~s}$ & $6.468596630947034 \mathrm{e}-07$ \\
$1.10 \mathrm{~s}$ & $7.800238491287989 \mathrm{e}-07$ \\
$1.20 \mathrm{~s}$ & $9.245426633263189 \mathrm{e}-07$ \\
$1.30 \mathrm{~s}$ & $1.0830954084400757 \mathrm{e}-06$ \\
$1.40 \mathrm{~s}$ & $1.2536181134137052 \mathrm{e}-06$ \\
\hline \hline
\end{tabular}

\section{Numerical experiments: Conical shells}

We conduct our second set of numerical tests in the case where the middle surface of the flexural shell under consideration is a portion of a cone (cf. Figure 4). 
e consider a domain $\omega$ shaped as follows:

$$
\omega:=\left\{\left(y_{1}, y_{2}\right) \in \mathbb{R}^{2} ; 0<y_{1}<\pi \text { and } 0.4<y_{2}<1\right\},
$$

where $\gamma_{0}$, the region of the boundary at which the clamping occurs, takes the form

$$
\gamma_{0}:=\left\{\left(y_{1}, y_{2}\right) \in \mathbb{R}^{2} ; y_{1}=\pi, y_{2} \in[0.4,1]\right\} .
$$

In curvilinear coordinates (see 22 and also Chapter 8 of [40]), the middle surface is given by the mapping $\boldsymbol{\theta}$ defined by

$$
\boldsymbol{\theta}\left(y_{1}, y_{2}\right)=\left(b y_{2} \cos y_{1}, b y_{2} \sin y_{1}, c y_{2}\right) \text {, }
$$

where $b>0$ and $c>0$.

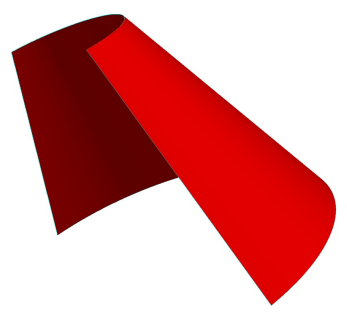

Figure 4. A conical shell.

This means that the vanishing displacement field is contained in one generatrix of the conical shell. As initial conditions in Problem $\mathcal{P}_{F}^{\varepsilon}(\omega)$, we take $\boldsymbol{\zeta}_{0}=\boldsymbol{\zeta}_{1}=\mathbf{0}$.

Then the covariant basis of the tangent plane to $\boldsymbol{\theta}(\bar{\omega})$ at the point $\boldsymbol{\theta}\left(y_{1}, y_{2}\right)$ is given by

$$
\begin{aligned}
& \boldsymbol{a}_{1}=\left(-b y_{2} \sin y_{1}, b y_{2} \cos y_{1}, 0\right), \\
& \boldsymbol{a}_{2}=\left(b \cos y_{1}, b \sin y_{1}, c\right), \\
& \boldsymbol{a}_{3}=\boldsymbol{a}^{3}=\left(\frac{c \cos y_{1}}{\sqrt{b^{2}+c^{2}}}, \frac{c \sin y_{1}}{\sqrt{b^{2}+c^{2}}}, \frac{-b}{\sqrt{b^{2}+c^{2}}}\right) .
\end{aligned}
$$

The covariant components of metric tensors on $\boldsymbol{\theta}(\bar{\omega})$ are given by

$$
a_{11}=\boldsymbol{a}_{1} \cdot \boldsymbol{a}_{1}=b^{2} y_{2}{ }^{2}, \quad a_{21}=a_{12}=0, \quad a_{22}=b^{2}+c^{2}
$$

and

$$
a=\operatorname{det}\left(a_{\alpha \beta}\right)=b^{2} y_{2}{ }^{2}\left(b^{2}+c^{2}\right) .
$$

The contravariant components of the first fundamental form of $\boldsymbol{\theta}(\bar{\omega})$ are given by

$$
a^{11}=\frac{1}{b^{2} y_{2}^{2}}, \quad a^{21}=a^{12}=0, \quad a^{22}=\frac{1}{b^{2}+c^{2}},
$$

Prepared using sagej.cls 
so that we obtain the following

$$
\begin{aligned}
& \boldsymbol{a}^{1}=a^{11} \boldsymbol{a}_{1}=\left(\frac{-\sin y_{1}}{b y_{2}}, \frac{\cos y_{1}}{b y_{2}}, 0\right), \\
& \boldsymbol{a}^{2}=a^{22} \boldsymbol{a}_{2}=\left(\frac{b \cos y_{1}}{b^{2}+c^{2}}, \frac{b \sin y_{1}}{b^{2}+c^{2}}, \frac{c}{b^{2}+c^{2}}\right) .
\end{aligned}
$$

Since,

$$
\begin{aligned}
& \partial_{1} \boldsymbol{a}_{1}=\left(-b y_{2} \cos y_{1},-b y_{2} \sin y_{1}, 0\right), \\
& \partial_{2} \boldsymbol{a}_{1}=\partial_{1} \boldsymbol{a}_{2}=\left(-b \sin y_{1}, b \cos y_{1}, 0\right), \\
& \partial_{2} \boldsymbol{a}_{2}=(0,0,0),
\end{aligned}
$$

then the covariant and mixed components of the second fundamental form of $\boldsymbol{\theta}(\bar{\omega})$ are given by

$$
\begin{aligned}
& b_{11}=\frac{-b c y_{2}}{\sqrt{b^{2}+c^{2}}}, \quad b_{12}=b_{21}=b_{22}=0, \\
& b_{1}^{1}=\frac{-c}{b y_{2} \sqrt{b^{2}+c^{2}}}, \quad b_{1}^{2}=b_{2}^{1}=b_{2}^{2}=0 .
\end{aligned}
$$

Then the Christoffel symbols of $\boldsymbol{\theta}(\bar{\omega})$ are given by

$$
\Gamma_{11}^{2}=\frac{-b^{2} y_{2}}{b^{2}+c^{2}}, \quad \Gamma_{12}^{1}=\Gamma_{21}^{1}=y_{2}{ }^{-1}, \quad \Gamma_{11}^{1}=\Gamma_{12}^{2}=\Gamma_{21}^{2}=\Gamma_{22}^{1}=\Gamma_{22}^{2}=0
$$

We take the material parameters including Young's modulus, Poisson ratio and density like those in Section 7 , We take $b=0.20 \mathrm{~m}$ and $c=0.40 \mathrm{~m}$.

We implement Newmark's scheme (4)- (5) by means of the software FreeFem++ (cf. [38]) and we visualise the results using ParaView (cf.[39]). In our experiments we use a mesh made of 4,500 elements, as displayed in Figure 5

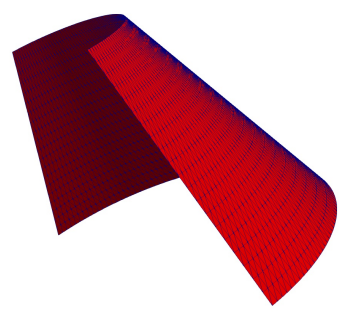

Figure 5. Mesh of the conical surface $\boldsymbol{\theta}(\bar{\omega})$ made of 4,500 elements.

Prepared using sagej.cls 
For conducting the numerical tests outputting Figure 6, we make use of the following announced parameters:

$$
\begin{aligned}
b & =0.20 \mathrm{~m}, \\
c & =0.40 \mathrm{~m}, \\
\varepsilon & =0.002 \mathrm{~m}, \\
E & =2.1 \times 10^{11} \mathrm{~Pa}, \\
\nu & =0.3, \\
\rho & =7.85 \times 10^{3} \mathrm{~kg} / \mathrm{m}^{3}, \\
\kappa & =10^{-6}, \\
\gamma & =0.6, \\
\beta & =\frac{(1 / 2+\gamma)^{2}}{4} .
\end{aligned}
$$

We consider the following dynamic loads

$$
\begin{aligned}
& p^{1, \varepsilon}\left(t, y_{1}, y_{2}\right)=10 t, \\
& p^{2, \varepsilon}\left(t, y_{1}, y_{2}\right)=\exp (t), \\
& p^{3, \varepsilon}\left(t, y_{1}, y_{2}\right)=\sin (t) .
\end{aligned}
$$

The deformations of the shell at different times are shown in Figure 6 for a time-step $\Delta t=0.01 \mathrm{~s}$. We observe that the clamping occurs at the left generatrix of the cylinder.

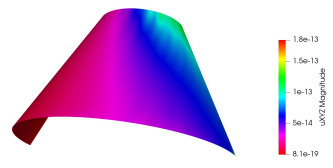

(a) $t=0.00 \mathrm{~s}$

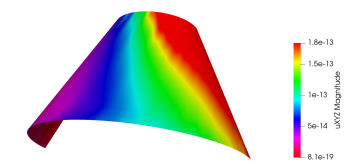

(d) $t=0.30 \mathrm{~s}$
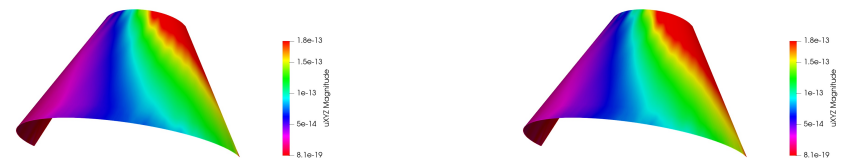

(b) $t=0.10 \mathrm{~s}$

(c) $t=0.20 \mathrm{~s}$

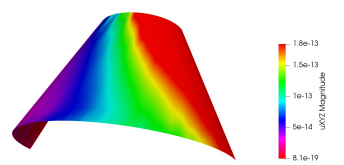

Prepared using sagej.cls 

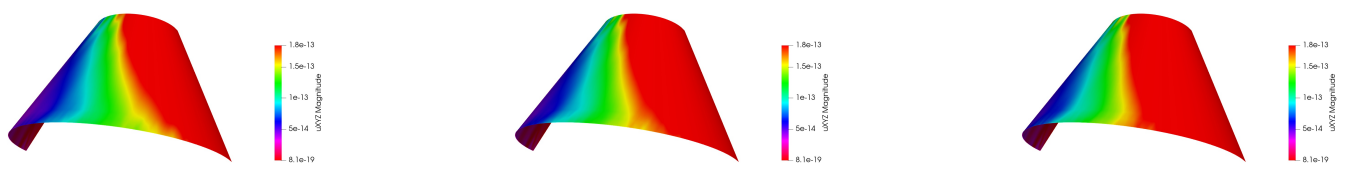

(g) $t=0.60 \mathrm{~s}$

(h) $t=0.70 \mathrm{~s}$

(i) $t=0.80 \mathrm{~s}$
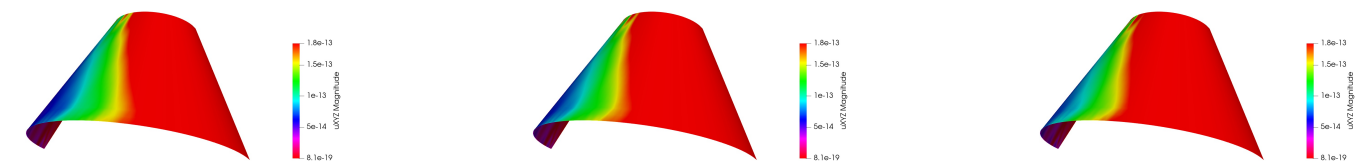

(j) $t=0.90 \mathrm{~s}$

(k) $t=1.00 \mathrm{~s}$

(I) $t=1.10 \mathrm{~s}$

Figure 6. $\mathrm{n}=150, \mathrm{~m}=30, \Delta t=0.01$.

\section{Final remarks: numerical experiments for the elliptic membrane shells model}

We conduct our third, and last, set of numerical tests in the case where the linearly elastic shell under consideration is an elliptic membrane shell (cf., Section 7 of 24]).

Following the terminology proposed in Section 4.1 of [21, such a shell is said to be an elliptic membrane shell if the following two additional assumptions are satisfied: first, $\gamma_{0}=\gamma$, i.e., the homogeneous boundary condition of place is imposed over the entire lateral face $\gamma \times[-\varepsilon, \varepsilon]$ of the shell, and second, its middle surface $\boldsymbol{\theta}(\bar{\omega})$ is elliptic, according to the definition given in Section 2 . Note that the assumption $\gamma_{0}=\gamma$ implies that the space $\boldsymbol{V}_{K}(\omega)$ introduced in Section 3 now reduces to

$$
\boldsymbol{V}_{K}(\omega)=H_{0}^{1}(\omega) \times H_{0}^{1}(\omega) \times H_{0}^{2}(\omega) .
$$

To begin with, we recall a crucial inequality that holds for elliptic surfaces (cf., e.g., Theorem 2.7-3 of [21]).

Theorem 9.1. Let $\omega$ be a domain in $\mathbb{R}^{2}$ and let $\boldsymbol{\theta} \in \mathcal{C}^{3}\left(\bar{\omega} ; \mathbb{E}^{3}\right)$ be an immersion such that $\boldsymbol{\theta}(\bar{\omega})$ is an elliptic surface. Define the space

$$
\boldsymbol{V}_{M}(\omega):=H_{0}^{1}(\omega) \times H_{0}^{1}(\omega) \times L^{2}(\omega),
$$

and the norm $\|\cdot\|_{\boldsymbol{V}_{M}(\omega)}$ by

$$
\|\boldsymbol{\eta}\|_{\boldsymbol{V}_{M}(\omega)}:=\left\{\sum_{\alpha}\left\|\eta_{\alpha}\right\|_{1, \omega}^{2}+\left\|\eta_{3}\right\|_{0, \omega}^{2}\right\}^{1 / 2} \quad \text { for each } \boldsymbol{\eta}=\left(\eta_{i}\right) \in \boldsymbol{V}_{M}(\omega)
$$

Prepared using sagej.cls 
Then there exists a constant $c=c(\omega, \boldsymbol{\theta})>0$ such that

$$
\|\boldsymbol{\eta}\|_{\boldsymbol{V}_{M}(\omega)} \leq c\left\{\sum_{\alpha, \beta}\left\|\gamma_{\alpha \beta}(\boldsymbol{\eta})\right\|_{0, \omega}^{2}\right\}^{1 / 2}
$$

for all $\boldsymbol{\eta}=\left(\eta_{i}\right) \in \boldsymbol{V}_{M}(\omega)$.

A natural formulation of a set of time-dependent two-dimensional equations (again, "twodimensional", in the sense that they are posed over the two-dimensional subset $\omega$ ) can be derived in the same way as in Section 3 .

Let us introduce the problem $\mathcal{P}_{M}^{\varepsilon}(\omega)$, describing the dynamics of an elliptic membrane shell.

Problem $\mathcal{P}_{M}^{\varepsilon}(\omega)$. Find a vector field $\boldsymbol{\zeta}^{\varepsilon}=\left(\zeta_{i}^{\varepsilon}\right):[0, T] \rightarrow \boldsymbol{V}_{M}(\omega)$ such that

$$
\boldsymbol{\zeta}^{\varepsilon} \in \mathcal{C}^{0}\left([0, T] ; \boldsymbol{V}_{M}(\omega)\right) \cap \mathcal{C}^{1}\left([0, T] ; \boldsymbol{L}^{2}(\omega)\right),
$$

that satisfies the following variational equations

$$
2 \varepsilon \rho \frac{\mathrm{d}^{2}}{\mathrm{~d} t^{2}} \int_{\omega} \zeta_{i}^{\varepsilon}(t) \eta_{i} \sqrt{a} \mathrm{~d} y+\varepsilon \int_{\omega} a^{\alpha \beta \sigma \tau} \gamma_{\sigma \tau}\left(\boldsymbol{\zeta}^{\varepsilon}(t)\right) \gamma_{\alpha \beta}(\boldsymbol{\eta}) \sqrt{a} \mathrm{~d} y=\int_{\omega} p^{i, \varepsilon}(t) \eta_{i} \sqrt{a} \mathrm{~d} y,
$$

for all $\boldsymbol{\eta}=\left(\eta_{i}\right) \in \boldsymbol{V}_{M}(\omega)$, in the sense of distributions in $(0, T)$, and that satisfies the following initial conditions

$$
\left\{\begin{array}{l}
\boldsymbol{\zeta}^{\varepsilon}(0)=\zeta_{0}, \\
\dot{\zeta}^{\varepsilon}(0)=\zeta_{1},
\end{array}\right.
$$

where $\boldsymbol{\zeta}_{0} \in \boldsymbol{V}_{M}(\omega)$ and $\boldsymbol{\zeta}_{1} \in \boldsymbol{L}^{2}(\omega)$ are prescribed.

We say that $\boldsymbol{\zeta}^{\varepsilon}$ is a strong solution of Problem $\mathcal{P}_{M}^{\varepsilon}(\omega)$ if

$$
\boldsymbol{\zeta}^{\varepsilon} \in \mathcal{C}^{0}\left([0, T] ; \boldsymbol{V}_{M}(\omega)\right) \cap \mathcal{C}^{1}\left([0, T] ; \boldsymbol{L}^{2}(\omega)\right),
$$

if $\boldsymbol{\zeta}^{\varepsilon}$ satisfies the variational equations of Problem $\mathcal{P}_{M}^{\varepsilon}(\omega)$ in the sense of distributions in $(0, T)$, and also satisfies the initial conditions.

The existence and uniqueness result is then classically established (cf., e.g., Theorem 8.2-2 of [34] and Theorem 7.2 of [24]).

Theorem 9.2. Problem $\mathcal{P}_{M}^{\varepsilon}(\omega)$ admits a unique strong solution $\boldsymbol{\zeta}^{\varepsilon} \in \mathcal{C}^{0}\left([0, T] ; \boldsymbol{V}_{M}(\omega)\right) \cap$ $\mathcal{C}^{1}\left([0, T] ; \boldsymbol{L}^{2}(\omega)\right)$.

We consider a linearly elastic elliptic membrane shell whose middle surface is a spherical cap of the unit sphere. The basis radius of the selected spherical cap is denoted by $r$ and, in general, such a quantity $r$ must be less or equal than the radius of the sphere which is here taken for constructing the spherical cap. In particular, in the case of the unit sphere, it must be $r \leq 1$.

The circumference constituting the basis of the spherical cap is parametrised as follows

$$
\left\{\begin{array}{l}
y_{1}=r \cos (t), \\
y_{2}=r \sin (t),
\end{array} \quad 0 \leq t \leq 2 \pi .\right.
$$

The spherical cap surface is then parametrised as follows

$$
\boldsymbol{\theta}\left(y_{1}, y_{2}\right)=\left(r y_{1}, r y_{2}, \sqrt{1-y_{1}^{2}-y_{2}^{2}}-\sin (\arccos (r))\right),
$$

Prepared using sagej.cls 
for all $\left(y_{1}, y_{2}\right)$ such that $\sqrt{y_{1}^{2}+y_{2}^{2}} \leq 1$.

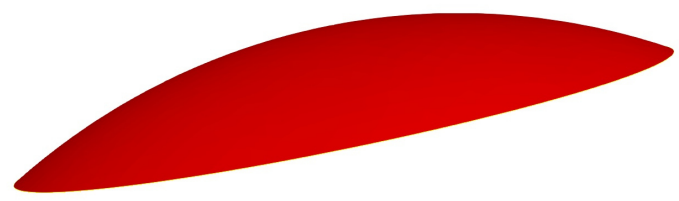

Figure 7. A spherical cap.

Let us recall that, for elliptic membrane shells, we have that the clamping occurs along the entire lateral boundary of the basis of the spherical cap. As initial conditions in Problem $\mathcal{P}_{M}^{\varepsilon}(\omega)$, we take $\zeta_{0}=\zeta_{1}=\mathbf{0}$.

Since the computation of the geometrical entities introduced in Section 1 involves a lot of machinery, we just limit ourselves to displaying the figures describing the evolution of the displacement magnitude over the middle surface under consideration. For performing the numerical experiments, we used the following parameters:

$$
\begin{aligned}
r & =0.50 \mathrm{~m}, \\
\varepsilon & =0.002 \mathrm{~m}, \\
E & =2.1 \times 10^{11} \mathrm{~Pa}, \\
\nu & =0.3, \\
\rho & =7.85 \times 10^{3} \mathrm{~kg} / \mathrm{m}^{3}, \\
\kappa & =10^{-6}, \\
\gamma & =0.6, \\
\beta & =\frac{(1 / 2+\gamma)^{2}}{4} .
\end{aligned}
$$

We implements Newmark's scheme by means of the software FreeFem++ (cf. e.g., [38]) and we visualise the results in ParaView (cf., e.g., [39]). We construct a mesh by relying on the potentialities of the software FreeFem++: More specifically, we indicate the compiler to divide the basis of the spherical cap into 100 arcs. The length of these arcs denotes the size of the mesh (see Figure 8).

Prepared using sagej.cls 


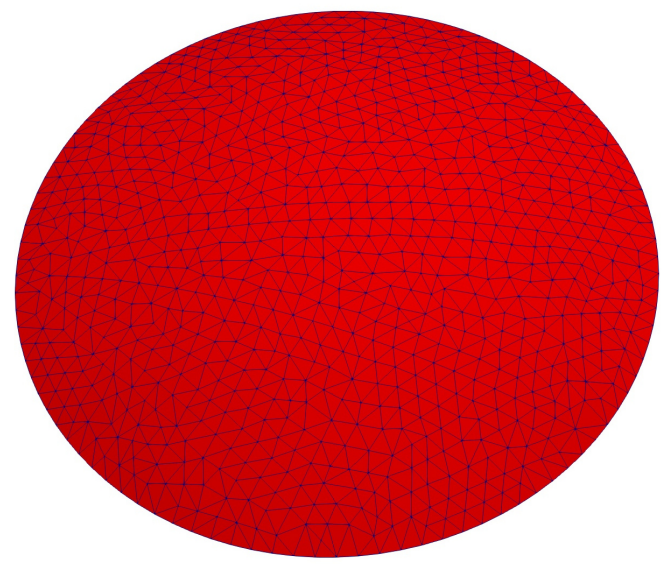

Figure 8. Spherical mesh constructed by relying on the potentialities of the software FreeFem ++ . The mesh size is obtained dividing by 100 the length of the circumference constituting the basis of the spherical cap.

We consider the following applied body force densities and surface force densities:

$$
\begin{aligned}
& p^{1, \varepsilon}\left(t, y_{1}, y_{2}\right)=0, \\
& p^{2, \varepsilon}\left(t, y_{1}, y_{2}\right)=0, \\
& p^{3, \varepsilon}\left(t, y_{1}, y_{2}\right)=\sin (t) .
\end{aligned}
$$

The deformations of the shell at different times are shown in Figure 9 for a time-step $\Delta t=0.01 \mathrm{~s}$. In the experiments we conducted, we applied a scaling factor of order 10,000 in ParaView, to visualise a more progressive evolution of the displacement field magnitude.

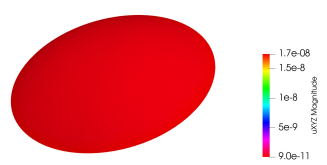

(a) $t=0.00 \mathrm{~s}$

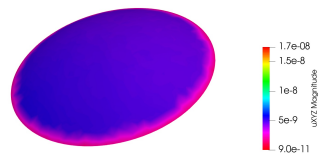

(b) $t=0.10 \mathrm{~s}$

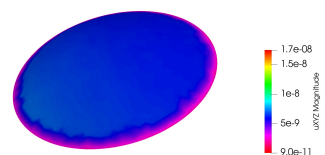

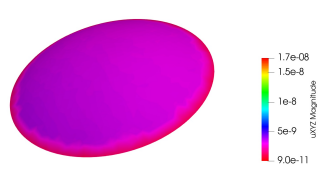

(c) $t=0.20 \mathrm{~s}$

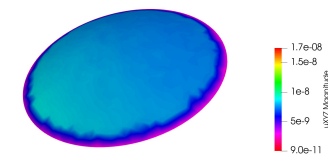

(f) $t=0.50 \mathrm{~s}$ 


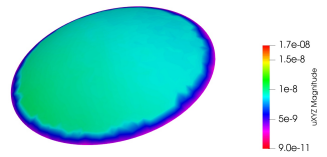

(g) $t=0.60 \mathrm{~s}$

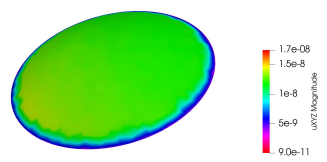

(j) $t=0.90 \mathrm{~s}$
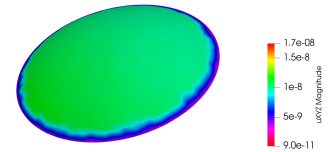

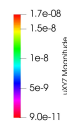

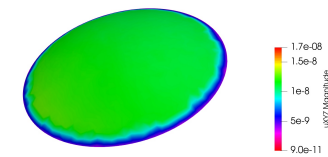

(h) $t=0.70 \mathrm{~s}$

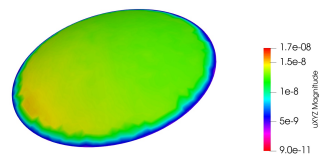

(k) $t=1.00 \mathrm{~s}$ (i) $t=0.80 \mathrm{~s}$

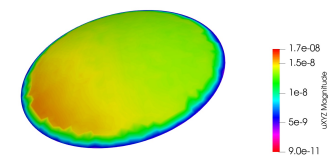

(I) $t=1.10 \mathrm{~s}$

Figure 9. $\mathrm{n}=150, \mathrm{~m}=30, \Delta t=0.01$.

\section{Acknowledgements}

The authors are very grateful to Professor Philippe G. Ciarlet for his encouragement and guidance.

The first author is supported by the National Natural Science Foundation of China (NSFC. 11971379, 11571275, 11572244), the Natural Science Foundation of Shaanxi Province (2018JM1014).

The third author acknowledges partial support by the ERC advanced grant 668998 (OCLOC) under the EU's H2020 research program.

\section{References}

[1] Huda MN, Jumat MZB and Islam ABMS. Flexural performance of reinforced oil palm shell and palm oil clinker concrete (pscc) beam. Constr Bulld Mater 2016; 127: 18-25.

[2] Singh VK, Mahapatra TR and K PS. Nonlinear flexural analysis of single/doubly curved smart composite shell panels integrated with pfrc actuator. Eur J Mech A-Solid 2016; 60: 300-314.

[3] Kar VR and Panda SK. Nonlinear flexural vibration of shear deformable functionally graded spherical shell panel. Compos Struct 2015; 18(3): 693-709.

[4] Totaro G. Flexural, torsional, and axial global stiffness properties of anisogrid lattice conical shells in composite material. Compos Struct 2016; 153: 738-745.

[5] Jayaprithika A and Sekar SK. Stress-strain characteristics and flexural behaviour of reinforced eco-friendly coconut shell concrete. Constr Build Mater 2016; 117: 244-250.

[6] Ciarlet PG and Destuynder P. A justification of the two-dimensional linear plate model. J Mécanique 1979; 18: 315-344. 
[7] Ciarlet PG and Miara B. Justification of a two-dimensional shallow shell model in linearized elasticity. C R Acad Sci Paris Sér I Math 1990; 311(9): 571-574.

[8] Ciarlet PG and Miara B. Justification of the two-dimensional equations of a linearly elastic shallow shell. Comm Pure Appl Math 1992; 45(3): 327-360.

[9] Ciarlet PG and Lods V. On the ellipticity of linear membrane shell equations. J Math Pures Appl 1996; 75: 107-124.

[10] Ciarlet PG and Lods V. Asymptotic analysis of linearly elastic shells. I. Justification of membrane shell equations. Arch Rational Mech Anal 1996; 136(2): 119-161.

[11] Ciarlet PG and Lods V. Asymptotic analysis of linearly elastic shells. III. Justification of Koiter's shell equations. Arch Rational Mech Anal 1996; 136(2): 191-200.

[12] Ciarlet PG and Lods V. Asymptotic analysis of linearly elastic shells: "generalized membrane shells". J Elasticity 1996; 43(2): 147-188.

[13] Ciarlet PG, Lods V and Miara B. Asymptotic analysis of linearly elastic shells. II. Justification of flexural shell equations. Arch Rational Mech Anal 1996; 136(2): 163-190.

[14] Ciarlet PG, Mardare C and Shen X. Donati compatibility conditions for membrane and flexural shells. Anal Appl 2015; 13(6): 685-705.

[15] Ciarlet PG, Mardare C and Piersanti P. An obstacle problem for elliptic membrane shells. Math Mech Solids 2019; 24(5): 1503-1529.

[16] Ciarlet PG, Mardare C and Piersanti P. Un problème de confinement pour une coque membranaire linéairement élastique de type elliptique. CR Acad Sci Paris, Sér I 2018; 356(10): 1040-1051.

[17] Ciarlet PG and Piersanti P. A confinement problem for a linearly elastic Koiter's shell. CR Acad Sci Paris, Sér I 2019; 357: 221-230.

[18] Ciarlet PG and Piersanti P. An obstacle problem for Koiter's shells. Math Mech Solids 2019; 24(10): 3061-3079.

[19] Xiao L. Asymptotic analysis of dynamic problems for linearly elastic shells-justification of equations for dynamic flexural shells. Chinese Ann Math Ser B 2001; 22(3): 13-22.

[20] Xiao L. Asymptotic analysis of dynamic problems for linearly elastic shells-justification of equations for dynamic Koiter shells. Chinese Ann Math Ser B 2001; 22(3): 267-274.

[21] Ciarlet PG. Mathematical Elasticity. Vol. III: Theory of Shells. Amsterdam: North-Holland, 2000.

[22] Ciarlet PG. An Introduction to Differential Geometry with Applications to Elasticity. Dordrecht: Springer, 2005.

[23] Leoni G. A First Course in Sobolev Spaces, Graduate Studies in Mathematics, volume 181. Second ed. Providence: American Mathematical Society, 2017.

Prepared using sagej.cls 
[24] Piersanti P. An existence and uniqueness theorem for the dynamics of flexural shells. Math Mech Solids accepted; .

[25] Ciarlet PG. Mathematical Elasticity. Vol. I: Three-Dimensional Elasticity. Amsterdam: NorthHolland, 1988.

[26] Koiter WT. On the foundations of the linear theory of thin elastic shells. I, II. Nederl Akad Wetensch Proc Ser B 73 (1970), 169-182; ibid 1970; 73: 183-195.

[27] Bernadou M and Ciarlet PG. Sur l'ellipticité du modèle linéaire de coques de W. T. Koiter. Computing Methods in Applied Sciences and Engineering, Part 1 1996; 134: pp. 89-136.

[28] Bernadou M, Ciarlet PG and Miara B. Existence theorems for two-dimensional linear shell theories. J Elasticity 1994; 34: 111-138.

[29] Lions JL. Quelques méthodes de résolution des problèmes aux limites non linéaires. Dunod; Gauthier-Villars, Paris, 1969.

[30] Brezzi F and Fortin M. Mixed and Hybrid Finite Element Methods. New York: Springer, 1991.

[31] Ciarlet PG. Introduction to Numerical Linear Algebra and Optimisation. Cambridge: Cambridge University Press, 1989.

[32] Ciarlet PG. The Finite Element Method for Elliptic Problems. Amsterdam: North-Holland, 1978.

[33] Brenner S and Scott LR. The mathematical theory of finite element methods. Third ed. New York: Springer, 2008.

[34] Raviart PA and Thomas JM. Introduction à l'Analyse Numérique des Équations aux Dérivées Partielles. Paris: Dunod, 1988.

[35] Quarteroni A and Valli A. Numerical Approximation of Partial Differential Equations. Berlin: Springer-Verlag, 2009.

[36] Newmark NM. A method of computation for structural dynamics. J Eng Mech-ASCE 1959; 85(EM3): 67-94.

[37] Goncalves PB, Silva FMA, Rega G et al. Global dynamics and integrity of a two-dof model of a parametrically excited cylindrical shell. Nonlinear Dynam 2011; 63(1): 61-82.

[38] Hecht F. New development in FreeFem ++. Numer Math 2012; 20(3-4): 251-265.

[39] Ahrens J, Geveci B and Law C. ParaView: An End-User Tool for Large Data Visualization. Elsevier: Visualization Handbook, 2005. ISBN-13: 978-0123875822.

[40] Ciarlet PG. Linear and Nonlinear Functional Analysis with Applications. Philadelphia: Society for Industrial and Applied Mathematics, 2013. 